ARTICLE

DOI: $10.1038 / s 41467-018-06965-w$

\title{
Arabidopsis AGDP1 links H3K9me2 to DNA methylation in heterochromatin
}

Cuijun Zhang1,2, Xuan Du1,3, Kai Tang (10 1,2, Zhenlin Yang 1,3, Li Pan ${ }^{4}$, Peipei Zhu4, Jinyan Luo, Yuwei Jiang ${ }^{1}$, Hui Zhang ${ }^{1}$, Huafang Wan ${ }^{2,6}$, Xingang Wang ${ }^{2}$, Fengkai Wu ${ }^{2,7}$, W. Andy Tao ${ }^{4}$, Xin-Jian He (1) ${ }^{5}$, Heng Zhang @ ${ }^{1}$, Ray A. Bressan ${ }^{2}$, Jiamu Du (i) ${ }^{1} \&$ Jian-Kang Zhu (i) ${ }^{1,2}$

Heterochromatin is a tightly packed form of chromatin that is associated with DNA methylation and histone 3 lysine 9 methylation (H3K9me). Here, we identify an H3K9me2binding protein, Agenet domain (AGD)-containing p1 (AGDP1), in Arabidopsis thaliana. Here we find that AGDP1 can specifically recognize the H3K9me2 mark by its three pairs of tandem AGDs. We determine the crystal structure of the Agenet domain 1 and 2 cassette (AGD12) of Raphanus sativus AGDP1 in complex with an H3K9me2 peptide. In the complex, the histone peptide adopts a unique helical conformation. AGD12 specifically recognizes the $\mathrm{H} 3 \mathrm{~K} 4 \mathrm{meO}$ and $\mathrm{H} 3 \mathrm{~K} 9 \mathrm{me} 2$ marks by hydrogen bonding and hydrophobic interactions. In addition, we find that AGDP1 is required for transcriptional silencing, non-CG DNA methylation, and H3K9 dimethylation at some loci. ChIP-seq data show that AGDP1 preferentially occupies long transposons and is associated with heterochromatin marks. Our findings suggest that, as a heterochromatin-binding protein, AGDP1 links H3K9me2 to DNA methylation in heterochromatin regions.

\footnotetext{
${ }^{1}$ National Key Laboratory of Plant Molecular Genetics, CAS Center for Excellence in Molecular Plant Sciences, Shanghai Center for Plant Stress Biology, Shanghai Institutes for Biological Sciences, Chinese Academy of Sciences, 201602 Shanghai, China. ${ }^{2}$ Department of Horticulture and Landscape Architecture, Purdue University, West Lafayette, IN 47907, USA. ${ }^{3}$ University of Chinese Academy of Sciences, 100049 Beijing, China. ${ }^{4}$ Department of Biochemistry, Purdue University, West Lafayette, IN 47907, USA. ${ }^{5}$ National Institute of Biological Sciences, 102206 Beijing, China. ${ }^{6}$ College of Agronomy and Biotechnology, Southwest University, 400715 Chongqing, China. ${ }^{7}$ Maize Research Institute, Sichuan Agricultural University, 611130 Chengdu, China. These authors contributed equally: Cuijun Zhang, Xuan Du, Kai Tang. Correspondence and requests for materials should be addressed to J.D. (email: jmdu@sibs.ac.cn) or to J.-K.Z. (email: jkzhu@sibs.ac.cn)
} 
n eukaryotic cells, DNA wraps around eight histone protein cores to form nucleosomes, which are further compacted into higher order structures known as chromatin. The chromatin can be divided into euchromatin, where DNA is accessible for transcription, and heterochromatin, where DNA is less accessible for transcription ${ }^{1}$. Modifications on the $\mathrm{N}$-terminal tails of histones, which includes methylation, acetylation, phosphorylation, ubiquitylation, sumoylation, and $\mathrm{ADP}$-ribosylation ${ }^{2}$, provide a mechanism for fine regulation of chromatin states and gene expression.

In plants, $\mathrm{H} 3 \mathrm{~K} 9 \mathrm{me} 2$ is enriched at constitutively silenced heterochromatin and is required for the silencing of transposable elements (TEs) and other repetitive $\mathrm{DNA}^{3-6}$. Besides $\mathrm{H} 3 \mathrm{~K} 9 \mathrm{me} 2$, DNA methylation is another gene-silencing epigenetic mark. In plants, DNA methylation occurs at all three sequence contexts: $\mathrm{CG}, \mathrm{CHG}$ ( $\mathrm{H}$ denotes $\mathrm{A}, \mathrm{T}$, or $\mathrm{C}$ ), and $\mathrm{CHH}^{7-9}$. The non-CG methylation (CHG and $\mathrm{CHH}$ ) is mainly distributed in heterochromatin region and is highly associated with $\mathrm{H} 3 \mathrm{~K} 9 \mathrm{me}^{6}$. In Arabidopsis, the $\mathrm{H} 3 \mathrm{~K} 9 \mathrm{me} 2$ mark is solely established by the H3K9 methyltransferases SUVH4 (or KRYPTONITE), SUVH5, and SUVH6, which can recognize methylated $\mathrm{DNA}^{5,10-14}$. In the suvh4/5/6 triple mutant, H3K9me2 levels are strongly reduced globally, and both $\mathrm{CHG}$ and $\mathrm{CHH}$ methylation are also reduced $^{5,14,15}$. Heterochromatic $\mathrm{CHG}$ and $\mathrm{CHH}$ methylation, in contrast, are created by the DNA methyltransferases CMT3 and CMT2, respectively, both of which can specifically read the histone $\mathrm{H} 3 \mathrm{~K} 9 \mathrm{me} 2$ mark $^{5,16}$. Therefore, the heterochromatic non-CG methylation and $\mathrm{H} 3 \mathrm{~K} 9 \mathrm{me} 2$ are linked together by a cyclic reinforce loop between the writers of non-CG DNA methylation and $\mathrm{H} 3 \mathrm{~K} 9 \mathrm{me} 2^{6,17}$. Whether there is any additional internal linkage between heterochromatic DNA methylation and $\mathrm{H} 3 \mathrm{~K} 9 \mathrm{me} 2$, however, remains unclear.

Histone modifications can be "read" by specific "reader" modules to translate the upstream histone mark signal to downstream effectors ${ }^{18}$. In plants, the histone mark readers always have some plant-specific features ${ }^{19}$. The "Royal Family" histone readers include several well-known reader domains, such as tudor, Pro-Trp-Trp-Pro (PWWP), chromo, malignant brain tumor (MBT), and agenet (AGD) domains ${ }^{20}$. Among them, the tudor domain has been extensively studied as a histone methylation reader that can recognize multiple histone methylation marks such as $\mathrm{H} 3 \mathrm{~K} 4 \mathrm{me} 3, \mathrm{H} 3 \mathrm{~K} 9 \mathrm{me} 3, \mathrm{H} 3 \mathrm{~K} 36 \mathrm{me}$, and H4K20me2/ 3 , as well as some histone arginine methylation marks ${ }^{21}$. AGD was initially defined as a type of plant-specific tudor domain but also exists in some animal proteins such as human Fragile $\mathrm{X}$ Mental Retardation Protein (FMRP) ${ }^{20}$. The tandem AGD of FMRP has been reported to bind to the H3K79me2 mark both in vivo and in vitro ${ }^{22,23}$. Although some plausible aromatic residues for the methyllysine binding have been implicated in the structure of FMRP, the molecular mechanism of the recognition of methylated histone by AGD is still unknown, due to a lack of any histone peptide complex structure ${ }^{24}$.

Here, we identify a previously uncharacterized H3K9me2binding protein, which was named AGD-containing protein 1 (AGDP1). The AGDP1 protein possesses three pairs of tandem AGDs (AGD12, AGD34, and AGD56), all of which can specifically recognize the $\mathrm{H} 3 \mathrm{~K} 9$ me 2 mark. Our biochemical and structural studies reveal the molecular basis for the specific recognition of the dual marks $\mathrm{H} 3 \mathrm{~K} 4 \mathrm{me} 0$ and $\mathrm{H} 3 \mathrm{~K} 9 \mathrm{me} 2$ by the three tandem AGD cassettes of AGDP1. We also find that AGDP1 is required for transcriptional silencing, $\mathrm{CHG}$ and $\mathrm{CHH}$ methylation, and H3K9 dimethylation at some loci. Genome-wide mapping of AGDP1-binding sites by chromatin immunoprecipitation (ChIP)-seq show that most AGDP1-binding sites are in TE regions and are associated with the heterochromatin mark H3K9me2. The enrichment of multiple H3K9me2-binding sites on a single AGDP1 protein provides a structural basis for the genome-wide localization of AGDP1 to the H3K9me2-enriched heterochromatin. Our studies identify an H3K9me2-binding protein that regulates heterochromatic DNA methylation and reveal the molecular basis for the specific recognition of H3K9me2 mark, thus establishing an additional linkage between $\mathrm{H} 3 \mathrm{~K} 9 \mathrm{me} 2$ and heterochromatic DNA methylation.

\section{Results}

Identification of AGDP1 as an $\mathrm{H} 3 \mathrm{~K} 9 \mathrm{me2}-\mathrm{binding}$ protein. To identify readers of $\mathrm{H} 3 \mathrm{~K} 9$ methylation marks, we incubated the nuclear extracts of Arabidopsis flowers with synthesized biotinylated $\mathrm{H} 3(1-21) \mathrm{K} 9 \mathrm{me} 1, \mathrm{H} 3(1-21) \mathrm{K} 9 \mathrm{me} 2, \mathrm{H} 3(1-21) \mathrm{K} 9 \mathrm{me} 3$, or H3 (1-21) peptides. The peptides and their captured proteins were affinity purified with streptavidin beads and were analyzed by mass spectrometry. Consistent with previous reports, we found that CMT3, CMT2, and ENHANCED DOWNY MILDEW 2 (EDM2) were present in the H3K9me1, H3K9me2, and $\mathrm{H} 3 \mathrm{~K} 9 \mathrm{me} 3$ pull-down products but not in $\mathrm{H} 3$ pull-down products (Supplementary Data 1) $5,16,25$. CHROMATIN REMODELING FACTOR 17 (CHR17) was present in all of the peptide pull-down products, indicating that $\mathrm{CHR} 17$ can bind to histone $\mathrm{H} 3$ tails with or without $\mathrm{H} 3 \mathrm{~K} 9 \mathrm{me}$ modifications. Interestingly, an AGDcontaining protein was identified from the H3K9me1, H3K9me2, and H3K9me3 pull-down samples but not from the H3 pull-down samples. This protein was subsequently named AGDP1 (Supplementary Data 1). AGDP1 possesses six AGDs that can be divided into three tandem AGD cassettes: AGD12, AGD34, and AGD56 (Fig. 1a). To map the histone-binding region on AGDP1, we performed isothermal titration calorimetry (ITC)-based binding assay. Intriguingly, all three tandem AGD cassettes (AGD12, AGD34, and AGD56) can specifically bind to the methylated $\mathrm{H} 3 \mathrm{~K} 9$ mark with preference on $\mathrm{H} 3 \mathrm{~K} 9 \mathrm{me} 2$ (Figs. 1b-d), suggesting that a single AGDP1 protein has multiple H3K9me2-binding sites. We further measured the binding between the full-length AtAGDP1 and different H3K9 methylated peptides. Consistently, the full-length AtAGDP1 also showed a preference toward $\mathrm{H} 3 \mathrm{~K} 9 \mathrm{me} 2$. This binding yields a binding stoichiometry of about 3 (Fig. 1e), confirming that a single AGDP1 protein can bind to three H3K9me2 tails.

Structure of AGD12 in complex with an $\mathrm{H} 3 \mathrm{~K} 9 \mathrm{me} 2$ peptide. To investigate the molecular mechanism of the recognition of H3K9me2 by AGDP1, we performed structural studies. The AtAGDP1 AGD12 only produced low-resolution crystals that were not suitable for structure determination. As an alternative, we successfully obtained high-quality crystals of AGD12 of the Raphanus sativus AGDP1 (RsAGDP1), which displays 83\% sequence identity to AtAGDP1 and which also specifically binds to the H3K9me2 mark (Fig. 1a and Supplementary Fig. 1a-c), in complex with an $\mathrm{H} 3(1-15) \mathrm{K} 9 \mathrm{me} 2$ peptide. The structure was determined at $1.9 \AA$ resolution (Fig. 1f, Supplementary Fig. 1d, and Supplementary Table 1). The AGD12 of RsAGDP1 adopts a classical tandem tudor-like conformation (Fig. 1f). The two AGDs (AGD1 and AGD2) display the canonical AGD/tudor family twisted $\beta$-barrel-like fold (Fig. 1f). The overall RsAGDP1 AGD12 structure resembles the reported structure of the tandem AGD of FMRP with a superimposition RMSD of $1.9 \AA$ (Supplementary Fig. 1e $)^{24}$.

The unique helical conformation of the $\mathrm{H} 3$ peptide. Interestingly, the $\mathrm{H} 3 \mathrm{~K} 9 \mathrm{me} 2$ peptide adopts a unique helical conformation with the four residues from $\mathrm{H} 3 \mathrm{~K} 4$ to $\mathrm{H} 3 \mathrm{~A} 7$ forming a short $\alpha$-helix (Fig. If and Supplementary Fig. 1d). In addition to the $\alpha$ helix featured main chain-main chain hydrogen bonds, the side 
a

AtAGDP1

b

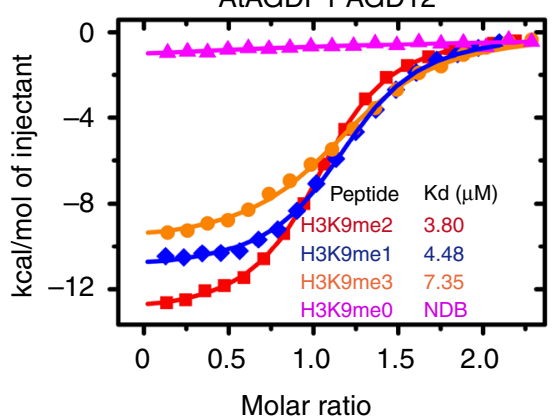

$c$

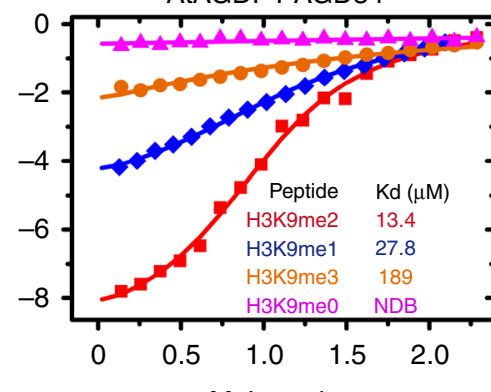

d

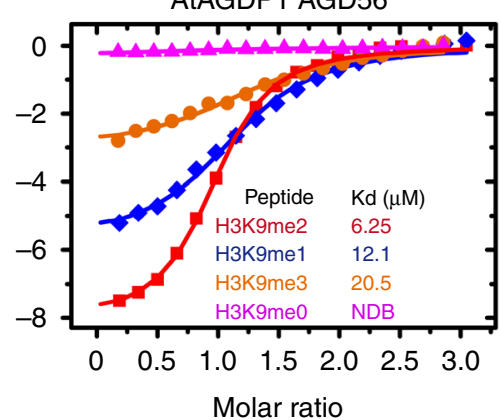

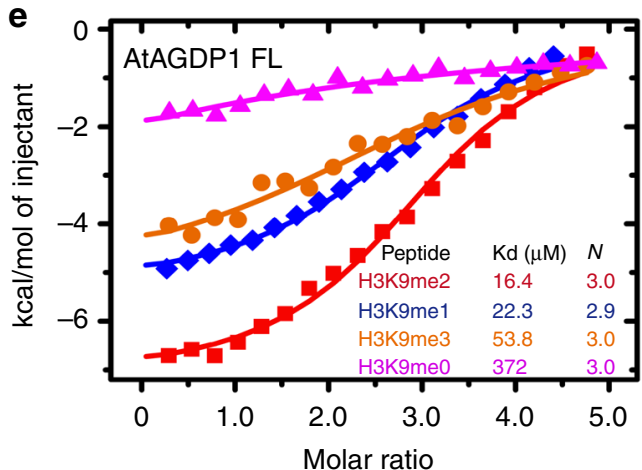

g

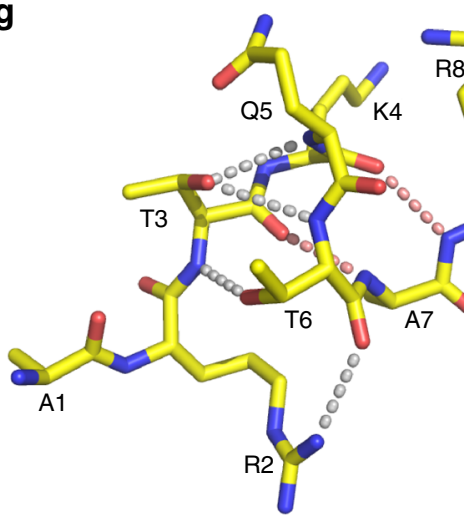

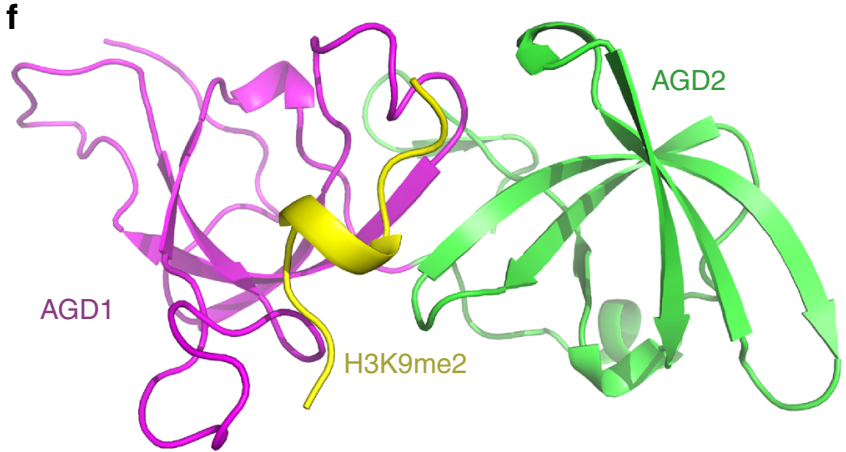

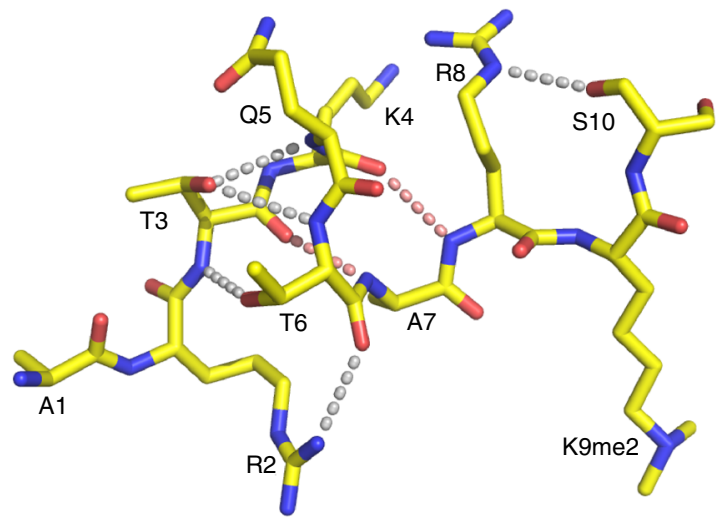

Fig. 1 Structure of the RsAGDP1 AGD12-H3K9me2 complex. a A schematic representation of the domain architecture of AtAGDP1 (upper panel), RsAGDP1 (middle panel), and the constructs of RsAGDP1 AGD12 and AtAGDP1 AGD34 used for crystallization in this research (lower panel). b-d ITC-binding curves between different methylated H3K9 peptides and AGD12 (b), AGD34 (c), and AGD56 (d) of AtAGDP1 show that all three tandem AGD cassettes recognize methylated $\mathrm{H} 3 \mathrm{~K} 9$ marks with a preference on $\mathrm{H} 3 \mathrm{~K} 9 \mathrm{me2}$. NDB, no detectable binding. e ITC-binding curves between different methylated H3K9 peptides and full-length AtAGDP1 show that the full-length AGDP1 recognize methylated H3K9 with a preference on $\mathrm{H} 3 \mathrm{~K} 9 \mathrm{me} 2$ and the bindings have a binding ratio near 3. FL, full-length. $\mathbf{f}$ The overall structure of the RsAGDP1 AGD12-H3K9me2 complex is shown in ribbon diagram with the AGD1, AGD2, and $\mathrm{H} 3 \mathrm{~K} 9$ me2 peptide colored in magenta, green, and yellow, respectively. The peptide adopts a unique helical conformation. $\mathbf{g} \mathrm{A}$ stereo view of the intramolecular hydrogen-bonding interactions within the H3K9me2 peptide. The main chain-main chain hydrogen bonds and side chain-main chain hydrogen bonds are highlighted by dashed red and silver lines, respectively 
chains of H3R2, H3T3, H3T6, and H3R8 also form extensive intra-molecular hydrogen-bonding interactions to stabilize the unique conformation of the peptide (Fig. $1 \mathrm{~g}$ ). The intra-molecular hydrogen bonds constrain the peptide into a contractive conformation that is distinct from the commonly observed $\beta$-strandlike or extended loop-like conformations ${ }^{18}$. This type of $\mathrm{H} 3$ tail conformation has been observed in the structure of Bromodomain Adjacent to Zinc Finger 2A (BAZ2A) PHD finger in complex with an $\mathrm{H} 3(1-10)$ peptide with the superimposition of the two peptide yielding an RMSD of $0.6 \AA$ (Supplementary Fig. 1f $)^{26}$, suggesting a possible common feature that enables the $\mathrm{H} 3 \mathrm{~N}$-terminus to adopt a helical conformation in binding to certain readers. We think that the unique helical conformation of the peptide may reduce the distance between its $\mathrm{N}$ - and C-termini and thus enable multiple interactions of the peptide by the reader over a short distance.

Molecular interactions between $\mathrm{H} 3 \mathrm{~K} 9 \mathrm{me} 2$ and AGD12. The AGD12 of RsAGDP1 employs a large negatively charged surface to accommodate the $\mathrm{H} 3$ peptide with the side chains of $\mathrm{H} 3 \mathrm{~K} 4$ and H3K9me2 inserting into two adjacent surface pockets of the AGD1 and AGD2, respectively (Figs. 2a, b), highlighting the important role of $\mathrm{H} 3 \mathrm{~K} 4$ and $\mathrm{H} 3 \mathrm{~K} 9 \mathrm{me} 2$ in the recognition. Other peptide residues also contribute to the interaction with the AGD12 of AGDP1. In detail, the H3A1 and H3R2 form main chain-main chain hydrogen bonds with the Lys81 and Phe78 of AGD1, respectively (Fig. 2c). The guanidino group of H3R2 forms hydrogen-bonding and salt bridge interactions with the Asp124 of AGD2 (Fig. 2c). The unmodified $\mathrm{H} 3 \mathrm{~K} 4$ forms hydrogen-bonding and salt bridge interactions with the acidic residues Glu89 and Glu45 from the AGD1, and Glu45 also interacts with the unmodified H3R8 (Fig. 2d). The side chain methyl group of H3A7 is accommodated by a shallow hydrophobic pocket formed by Phe48, Tyr53, and Leu77 via hydrophobic interactions (Fig. 2d). The dimethyllysine of H3K9me2 is accommodated by a classical aromatic cage formed by Tyr122, Trp127, and Phe144 of AGD2 by hydrophobic and cation- $\pi$ interactions like most reported methyllysine readers (Fig. 2e) ${ }^{18}$. In addition to the aromatic cage, a Glu149 residue can form a hydrogen bond with the dimethylammonium proton to strengthen the binding and facilitate the selectivity for dimethyllysine (Fig. 2e). The H3T3, H3Q5, H3T6, and H3S10 do not have specific interactions with the protein. We further performed structure-based mutagenesis studies. Except Y122A, which precipitated heavily, the mutations that disrupted the H3R2-, H3K4me0-, H3R8-, or H3K9me2-binding pocket significantly impaired the binding between AGD12 and the H3K9me2 peptide (Figs. 2f, g). We further investigated the influence of $\mathrm{H} 3 \mathrm{~K} 4$ methylation to the recognition by ITC. The H3K4me1K9me2 and H3K4me2K9me2 peptides show slightly decrease to the binding by AGD12 (Supplementary Fig. 1g), indicating that the tandem AGD of AGDP1 can biochemically tolerate the H3K4me1 and $\mathrm{H} 3 \mathrm{~K} 4 \mathrm{me} 2$. The H3K4me3K9me2 peptide displays a nearly fivefold decrease of the binding (Supplementary Fig. 1g), indicating that the higher H3K4 methylation state leads to a decreased binding by AGD12. Therefore, we conclude that AGD12 prefers an H3K4me0K9me2 state of the histone tail.

The peptide-binding interface of AGD12 is different from that of the SHH1 SAWADEE domain, indicating a different histonebinding interface on the tandem tudor-like domains (Fig. 2h). Note that all residues involved in the peptide recognition are strictly conserved among multiple plant species, including Arabidopsis thaliana, which was used for our functional analyses in this study (Supplementary Fig. 2).
Mechanism of the $\mathrm{H} 3 \mathrm{~K} 9 \mathrm{me} 2$ recognition by AGD34 and AGD56. We also performed structural studies on AGD34 and AGD56 of AtAGDP1. We obtained the free-form structure of AtAGDP1 AGD34 (Fig. 3a and Supplementary Table 1). The overall structure of AtAGDP1 AGD34 resembles that of RsAGDP1 AGD12 with all the peptide-binding residues, especially the $\mathrm{H} 3 \mathrm{~K} 4 \mathrm{me} 0$ - and $\mathrm{H} 3 \mathrm{~K} 9 \mathrm{me} 2$-binding residues, conserved and occupying the same positions (Fig. 3a). The structure-based sequence alignment of AtAGDP1 and RsAGDP1 tandem AGD cassettes indicates that most of the peptide-interacting residues, especially the $\mathrm{H} 3 \mathrm{~K} 4$ - and $\mathrm{H} 3 \mathrm{~K} 9 \mathrm{me} 2$-binding residues, are conserved (Fig. 3b). The RsAGDP1 Phe78 and Lys81 are not conserved. However, both Phe78 and Lys81 contribute to the interactions with AGDP1 by their main chains. Considering that our ITC data indicated that all three tandem AGD cassette have the same $\mathrm{H} 3 \mathrm{~K} 9 \mathrm{me}$ 2-binding preference, we conclude that the AGD12, AGD34, and AGD56 cassettes of AGDP1 share the same H3K9me2 recognition mechanism.

Structural basis for the preference of dimethyllysine. The tandem AGDs of AGDP1 bind to different methylated states of H3K9 with the strongest binding to dimethyllysine, lower binding to monomethyllysine and trimethyllysine, indicating a preference for dimethylation. In contrast, most of the reported methyllysine readers use the same aromatic cage to recognize the methyllysine with a preference for higher methylation states of trimethyllysine. To elucidate the molecular basis for this preference, we compared their aromatic cages. We used the $\mathrm{H} 3 \mathrm{~K} 9 \mathrm{me} 3$ preferred binding protein M-phase phosphoprotein 8 (MPP8) and Ubiquitin-like, Containing PHD and RING Finger Domains, 1 (UHRF1) as references ${ }^{27-30}$. MPP8 and UHRF1 have methyllysine-binding aromatic cages possessing relative open conformations (Figs. 3c, d). Whereas one side of the trimethyllysines is embedded in the aromatic cages, the other sides of the trimethyllysines are exposed (Figs. 3c, d). Although there are also negatively charged residues near the aromatic cages, they are aligned aside of the cage and leave the pocket open to accommodate bigger head group of the trimethyllysine (Figs. 3c, d) ${ }^{27-30}$. Therefore, we think that these negatively charged residues in MPP8 and UHRF1 mainly function in neutralizing the charge of the methyllysines. In contrast, RsAGDP1 AGD12 has a different type of aromatic cage that is similar to the $\mathrm{BAH}$ domain of mouse Origin of Replication Complex Subunit 1 (ORC1) (Figs. 3e, f) ${ }^{31}$. The aromatic residues are similar to MPP8 or UHRF1, but the negatively charged residues are located differently (Figs. 3c-f). In AGDP1 and ORC1, the aromatic cages are covered by the negatively charged residues, forming closed cages to accommodate the methyllysine (Figs. 3e, f) ${ }^{31}$. The negatively charged residues not only neutralize the charge of the methyllysines but also form direct hydrogen bonds with the free protons of the dimethyllysine, leading to a preference to the free proton containing dimethyllysine but not the trimethyllysine. In addition, the closed pockets of AGDP1 and ORC1 are too narrow to accommodate the trimethyllysine, resulting in the preference for the dimethyllysine and decreased binding to trimethyllysine. Although the monomethyllysine can also form a direct hydrogen bond with and can be accommodated by AGDP1/ORC1 type binding pocket, the single methyl group has an obviously decreased binding by the aromatic cage, resulting a weaker binding than the dimethyllysine. However, there are still minor differences between the tandem AGD cassettes. For example, the binding affinity AtAGD12 to H3K9me2 $(3.8 \mu \mathrm{M})$ is only 1.2 -fold higher than $\mathrm{H} 3 \mathrm{~K} 9 \mathrm{me} 1(4.48 \mu \mathrm{M})$ (Fig. 1b). In contrast, the AtAGD34 and AtAGD56 binds to H3K9me2 about twofold higher than H3K9me1 (Figs. 1c, d), although the peptide-binding regions are quite conserved 
a

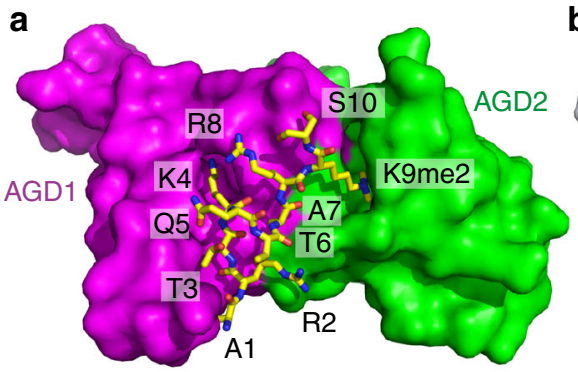

C

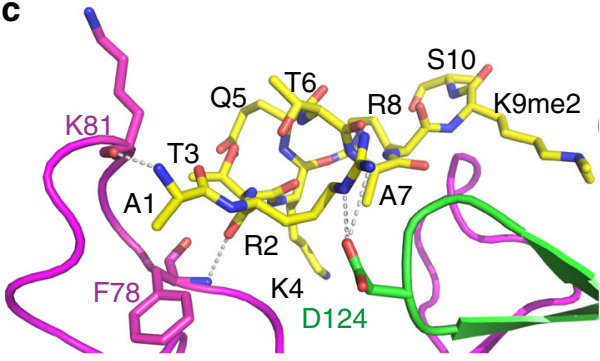

b
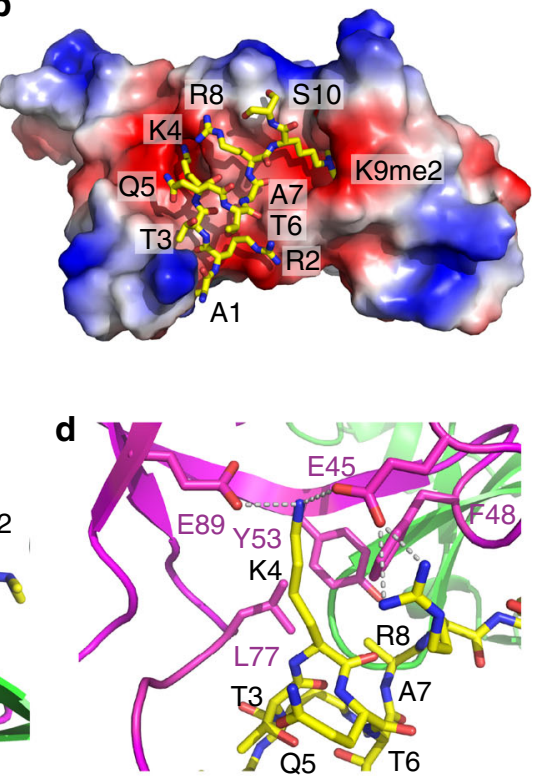

e

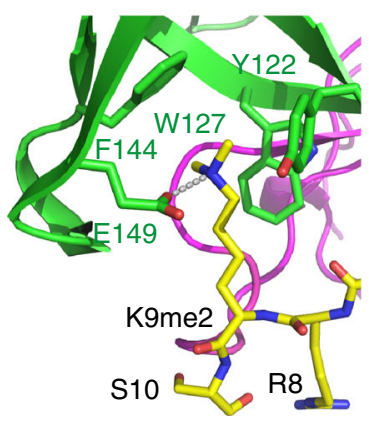

h

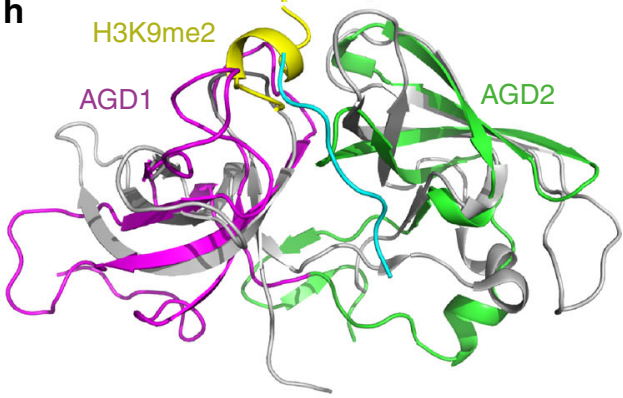

SAWADEE-H3K9me2 complex
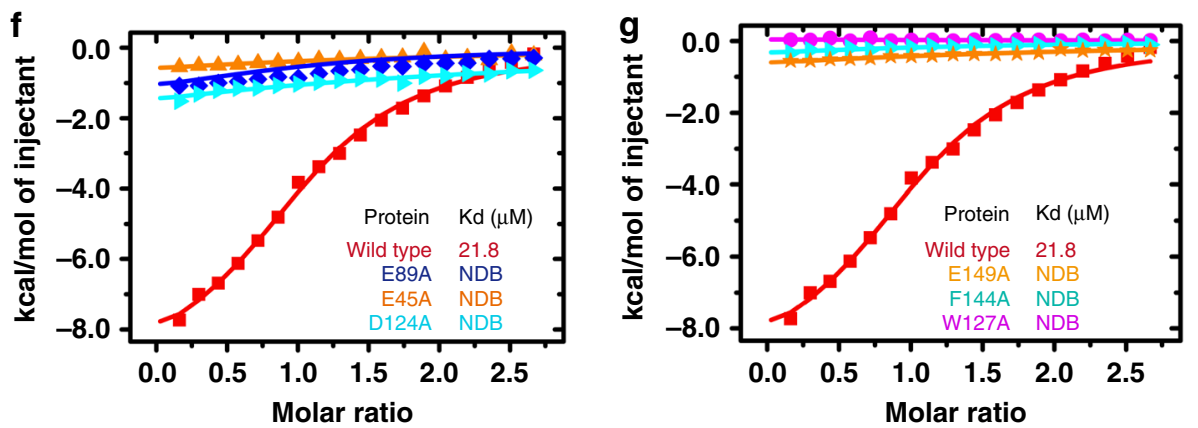

Fig. 2 Interactions between the H3K9me2 peptide and RsAGDP1 AGD12. a A surface view of the RsAGDP1 AGD12 with AGD1 and AGD2 colored in magenta and green, respectively. The $\mathrm{H} 3 \mathrm{~K} 9 \mathrm{me} 2$ peptide is shown in stick representation. The side chains of $\mathrm{H} 3 \mathrm{~K} 4 \mathrm{meO}$ and $\mathrm{H} 3 \mathrm{~K} 9 \mathrm{me} 2$ insert into two adjacent surface pockets on AGD1 and AGD2, respectively. b An electrostatics surface view of the RsAGDP1 AGD12 with the H3K9me2 peptide in stick representation. The peptide binds at a large negatively charged surface with $\mathrm{H} 3 \mathrm{~K} 4 \mathrm{meO}$ and $\mathrm{H} 3 \mathrm{~K} 9 \mathrm{me} 2$ inserting into two negatively charged surface pockets, respectively. c-e The detailed recognition of H3A1 and H3R2 (c), H3K4, H3A7, and H3R8 (d), and H3K9me2 (e). The interacting residues and the hydrogen bonds are highlighted in stick and dashed silver lines, respectively. f-g The ITC-binding curves between H3K9me2 and RsAGDP1 AGD12 mutations disrupting the H3R2, H3K4, and $\mathrm{H} 3 \mathrm{R} 8$ (f) or the $\mathrm{H} 3 \mathrm{~K} 9 \mathrm{me} 2(\mathbf{g})$ binding residues show that the mutations of critical residues significantly impair the binding between AGD12 and H3K9me2. $\mathbf{h}$ A superimposition of RsAGDP1 AGD12-H3K9me2 complex (in color) and SHH1 SAWADEE-H3K9me2 complex (in silver, PDB code: 4IUT). The H3K9me2 peptide from the SHH1 SAWADEE complex is colored in cyan. The AGDP1 AGD12 and SHH1 SAWADEE generally adopt a similar folding topology with their binding H3K9me2 peptides locating at the shorter and longer edge of the two AGD/tudor domain interface, respectively

(Fig. 3b). The relative preferences of $\mathrm{H} 3 \mathrm{~K} 9 \mathrm{me} 3$ and $\mathrm{H} 3 \mathrm{~K} 9 \mathrm{me} 1$ by different tandem AGD cassettes are different, too. A possible reason could be that some minor differences outside the H3K9me2-binding pocket may slightly change the shape and charge of the H3K9me2-binding pockets, leading to different sublevel preferences. These sub-level preferences may participate in the fine tuning functions of different tandem AGD cassettes and provide a plausible additional layer of regulation. 

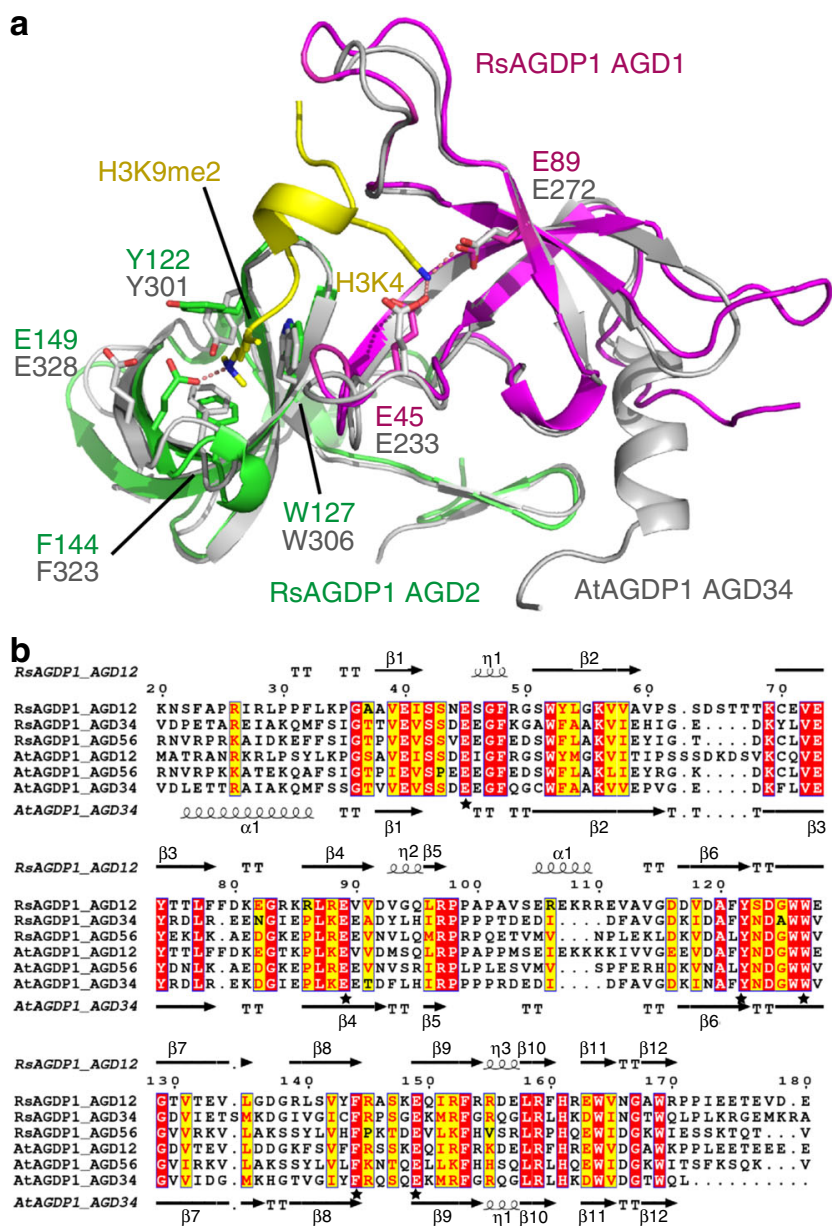

C

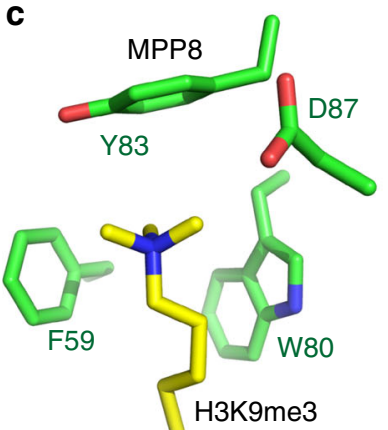

d
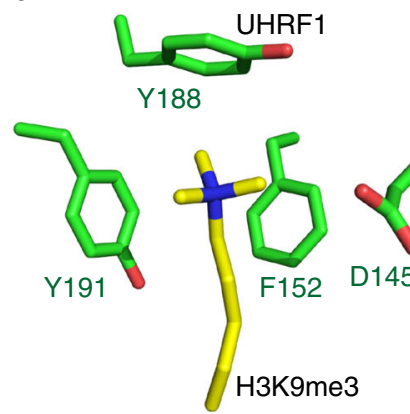

e

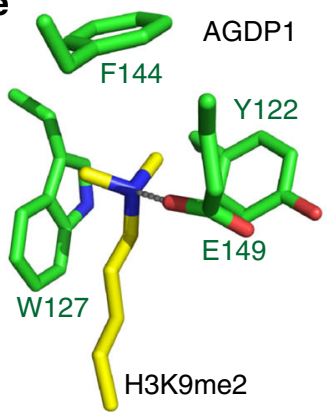

f

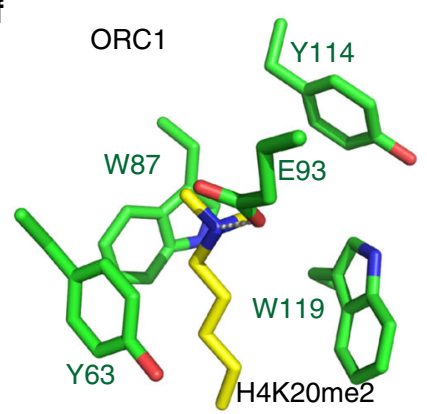

Fig. 3 AGD12, AGD34, and AGD56 of AGDP1 recognize methylated H3K9 by the same mechanism. a A superimposition of the structures of RsAGDP1 AGD12-H3K9me2 complex (in color) and AtAGDP1 AGD34 (in silver) indicate that the AGD12 and AGD34 adopt almost identical overall structures. The H3K4- and H3K9me2-binding residues are highlighted in sticks and they are conserved and occupy the same positions. $\mathbf{b} A$ structure-based sequence alignment of AtAGDP1 AGD12, AGD34, and AGD56, and RsAGDP1 AGD12, AGD34, and AGD56 with the secondary structures of RsAGDP1 AGD12 and AtAGDP1 AGD34 highlighted on the top and bottom of the alignment, respectively. The H3K4- and H3K9me2binding residues are highlighted by stars, and most of them are conserved. We therefore conclude that all the tandem AGD cassettes of AtAGDP1 and RsAGDP1 recognize H3K9me2 via a shared mechanism with conserved peptide-interacting residues. c-f The comparison of the methyllysinearomatic cage interaction of MPP8 (c, PDB code: 3R93), UHRF1 (d, PDB code: 4GY5), AGDP1 (e), and ORC1 (f, PDB code: 4DOW). The residues are shown in stick representation with the reader and methyllysine residues colored in green and yellow, respectively. The hydrogen bonds between dimethyllysine and the negatively charged residues are highlighted in dashed silver lines

developmental phenotypes for agdp1-1 and agdp1-2 mutants compared with the wild-type. Because AGDP1 can bind to the silencing mark $\mathrm{H} 3 \mathrm{~K} 9 \mathrm{me} 2$, we wondered whether AGDP1 is required for transcriptional silencing. We performed RNA deep sequencing assay with five replicates for wild-type and agdp1-1, and three replicates for suvh4/5/6. We found that only 15 TEs and 42 protein-coding genes $(P<0.05$; $\log 2$ (fold-change) $>1$; Cufflinks) were upregulated in the agdp1-1 mutants (Supplementary Data 2). The upregulated TEs were validated by quantitative reverse transcription (RT)-PCR analyses, which showed that 12 of them were upregulated in both agdp1-1 and agdp1-2 mutants (Fig. 4a). Ninety-one percent (11 of 12) of upregulated TEs and $57 \%$ (24 of 42 ) of upregulated protein-coding genes in agdp1-1 mutants were derepressed in suvh4/5/6 mutants (Supplementary Data 2), suggesting that some of the SUVH4/5/6-dependent loci are under regulation by AGDP1. We also determined the expression levels of the previously characterized hypermethylated loci AtMU1, AtGP1, AtSN1, and ERT7 by quantitative (RT)-PCR and found that these four loci were also upregulated in $\operatorname{agdp1-1}$ and agdp1-2 mutants (Fig. 4b). These four loci were not identified as upregulated TEs or genes by RNA-Seq, perhaps due to their relatively low expression levels. In any case, the results suggest that there may be additional loci that are regulated by AGDP1.

AGDP1 is required for both CHG and CHH methylation. To determine whether AGDP1 has a role in DNA methylation, we performed whole-genome bisulfite sequencing (WGBS) for the wild-type and agdp1-1 mutant plants. We identified 1456 CG, $9633 \mathrm{CHG}$, and $6204 \mathrm{CHH}$ hypomethylated differentially methylated regions (hypo-DMRs) in agdp1-1 compared with the wild-type; however, we identified only 1479 CG, $150 \mathrm{CHG}$, and $1939 \mathrm{CHH}$ hyper-DMRs in agdp1-1 (Fig. 4c and Supplementary Data 3 ). The large number of $\mathrm{CHG}$ and $\mathrm{CHH}$ hypo-DMRs in agdp1-1 suggested that AGDP1 is required for both CHG and $\mathrm{CHH}$ methylation in the genome. The $\mathrm{CHG}$ and $\mathrm{CHH}$ hypoDMRs are mainly located in pericentromeric heterochromatin regions (Fig. 4d). In addition, most of the $\mathrm{CHG}$ and $\mathrm{CHH}$ hypoDMRs are in TE regions (Fig. 4e). Through classification analysis for TE families, we found that DNA/En-Spm, LTR/Copia, LTR/ Gypsy are enriched in both CHG and CHH hypo-DMRs (Supplementary Data 4). Previously, Panda et al. divided TEs to truncated TEs and full-length $\mathrm{TEs}^{32}$. Compared with the truncated TEs, the full-length TEs are enriched in both CHG and

AGDP1 contributes to transcriptional silencing at some loci. To investigate the function of AGDP1, we obtained two individual T-DNA insertion mutants, agdp1-1 (Salk_134878) and agdp1-2 (Salk_130936), from ABRC (Supplementary Fig. 3a-c). Under normal growth conditions, we did not observe 


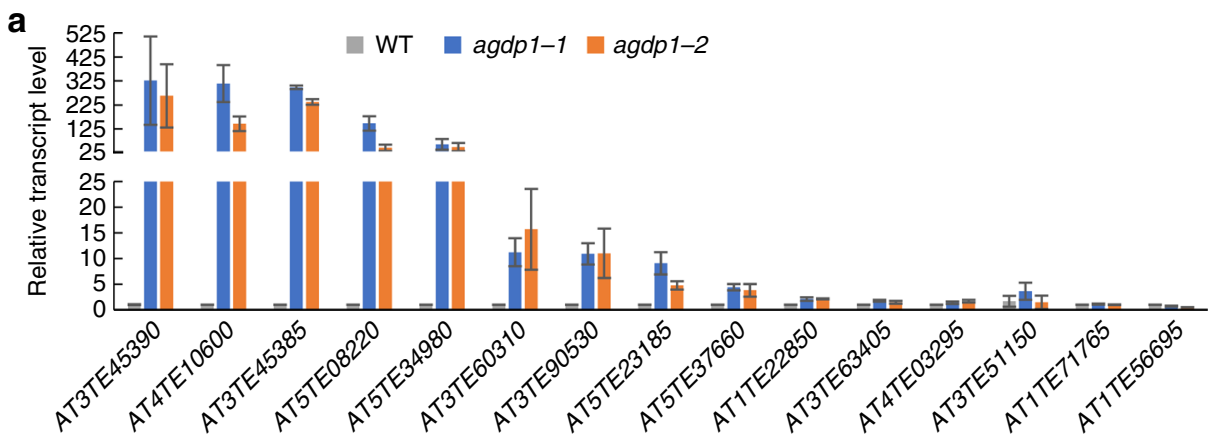

b

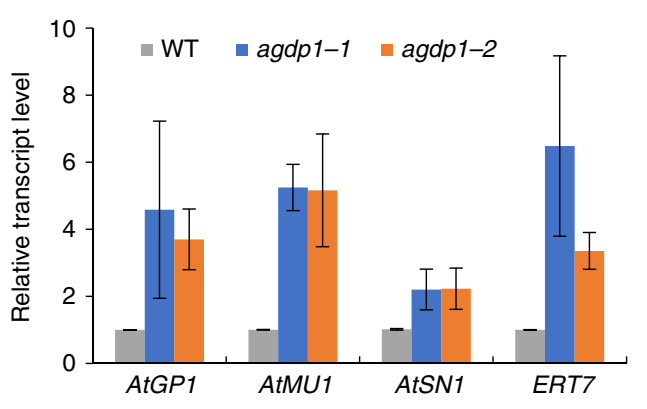

d

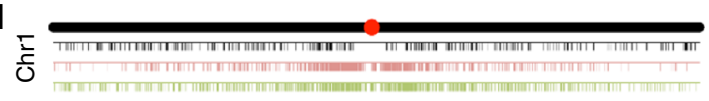

ㄴ.

仓

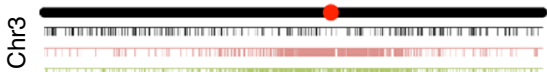

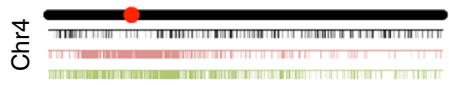

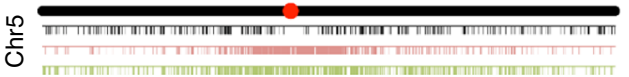

C

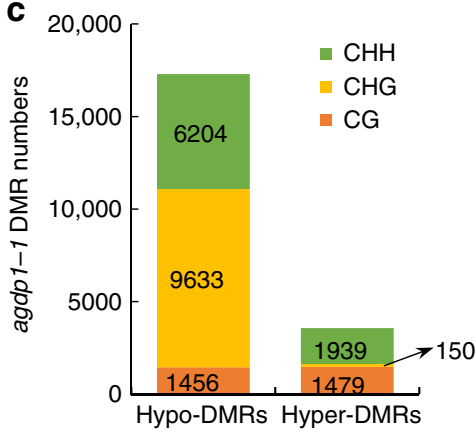

e

$\square$ TE $\square$ Gene $\square$ Intergenic - Other

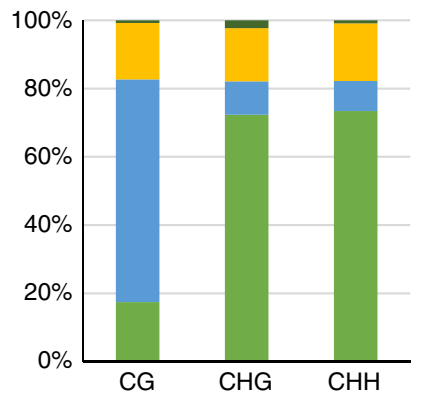

$\mathbf{f}$
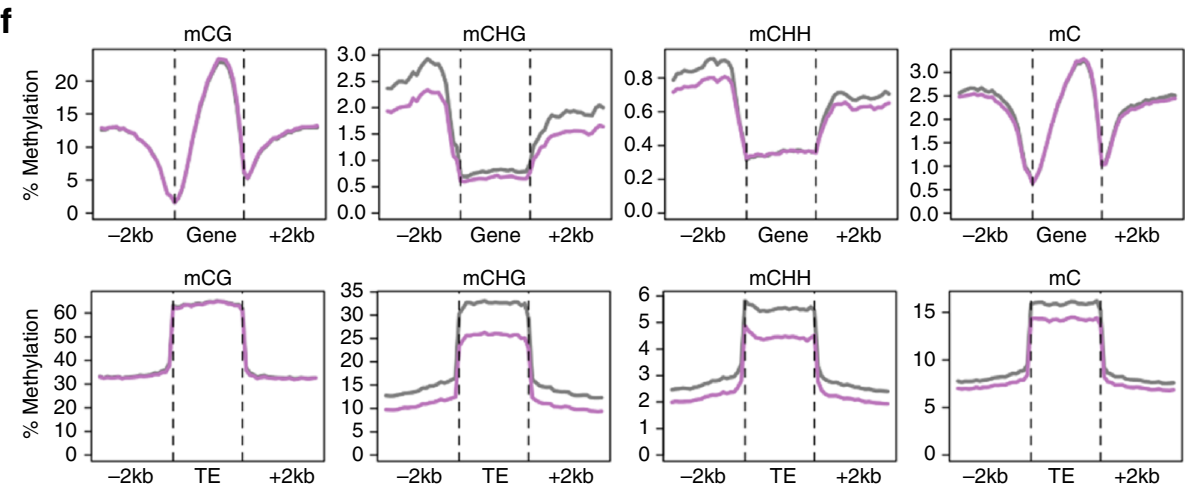

Fig. 4 AGDP1 contributes to transcriptional silencing and non-CG methylation at some loci. a The transcript levels of the indicated TEs were determined by quantitative RT-PCR in the wild-type, agdp1-1, and agdp1-2. Error bars are SDs of three biological replicates. b The RNA transcripts of AtMU1, AtGP1, AtSN1, and ERT7 were detected by quantitative RT-PCR in the wild-type, agdp1-1, and agdp1-2. Error bars are SDs of three biological replicates. c The numbers of CG, CHG, and CHH hypo-DMRs and hyper-DMRs identified in agdp1-1. d The distribution of hypo-DMRs throughout the five chromosomes. Black, pink, and green bars represent CG, CHG, and CHH hypo-DMRs, respectively. Red circles indicate centromeres. e Composition of the genomic location of the hypoDMRs. TE, transposable element. $\mathbf{f}$ Average DNA methylation levels along protein-coding genes (upper) and TEs (lower) and the 2-kb flanking regions in different cytosine contexts. Gray and pink curves represent DNA methylation levels of wild-type and agdp1-1, respectively 
$\mathrm{CHH}$ hypo-DMRs of agdp1-1 (Supplementary Data 4). By analyzing the average DNA methylation levels along protein-coding genes and TEs in different cytosine contexts, we found that CHG and $\mathrm{CHH}$ methylation are decreased in TE regions and in the 2$\mathrm{kb}$ flanking regions of protein-coding genes in the agdp1-1 mutant (Fig. 4f). In contrast, the CG methylation was not changed in protein-coding genes and TE regions in the agdp1-1 mutant, suggesting that agdp1 mainly affects $\mathrm{CHG}$ and $\mathrm{CHH}$ methylation. The reduction of DNA methylation in agdp1-1 and agdp1-2 mutants was confirmed by chop-PCR (Supplementary Fig. 3d).

agdp1 affects SUVH4/5/6-dependent $\mathrm{CHG}$ and $\mathrm{CHH}$ methylation. Both $\mathrm{CHG}$ and $\mathrm{CHH}$ methylation are reduced in the suvh4/5/6 triple mutant ${ }^{15}$. The finding that AGDP1 helps maintain $\mathrm{CHG}$ and $\mathrm{CHH}$ methylation levels in the genome led us to compare the hypo-DMRs of agdp1-1 and suvh4/5/6 mutants. We found that about $87 \%$ of agdp1-1 CHG-hypomethylated sites overlapped with suvh4/5/6 CHG-hypomethylated sites (Fig. 5a). For $\mathrm{CHH}$ methylation, $49 \%$ of agdp1-1 CHH-hypomethylated sites overlapped with suvh4/5/6 CHH-hypomethylated sites (Fig. 5a). Box plots analysis showed that the $\mathrm{CHG}$ and $\mathrm{CHH}$ methylation levels at the suvh4/5/6-specific loci also appeared decreased in the agdp1-1 mutant (Fig. 5a), although the decreases in agdp1-1 were insufficient to cause the loci to be considered hypo-DMRs. These results suggest that SUVH4/5/6-dependent CHG and $\mathrm{CHH}$ methylation are reduced in the agdp1-1 mutant.

To further analyze the effect of the agdp1-1 mutation on DNA methylation pathways, we performed hypo-DMR overlap analysis between agdp1-1 and $c m t 2, c m t 3, d r m 1 / 2$, or $d d m 1$ mutants with published WGBS data ${ }^{15,33}$. We found that most of the $\mathrm{CHG}$ hypo-DMRs of agdp1-1 overlapped with cmt3 and $d d m 1$ (Supplementary Fig. 4). For the CHH context, 45\%, 28\%, and 54\% of the agdp1-1 CHH-hypomethylated sites overlapped with $c m t 2, d r m 1 / 2$, and $d d m 1$, respectively (Supplementary Fig. 4). Consistent with the overlap analysis, box plot analysis showed that at agdp1-1 CHG hypo-DMRs, there was a strong loss of CHG methylation in suvh $4 / 5 / 6, c m t 3$, and $d d m 1$ mutants (Supplementary Fig. 5). At agdp1-1 CHH hypo-DMRs, the CHH methylation a
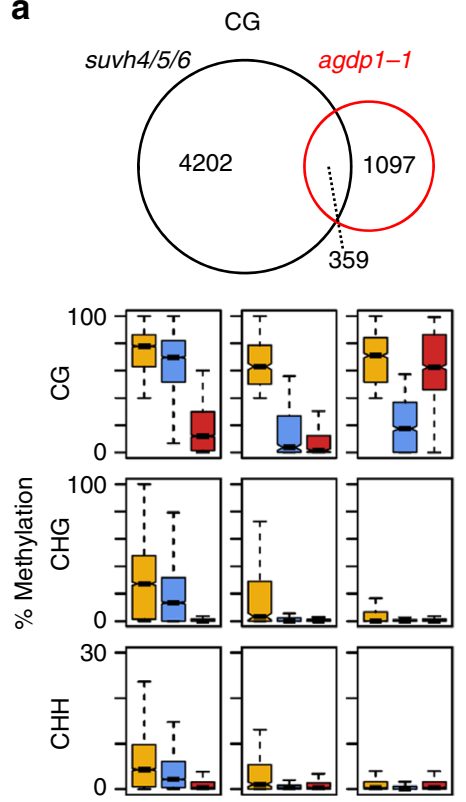

b

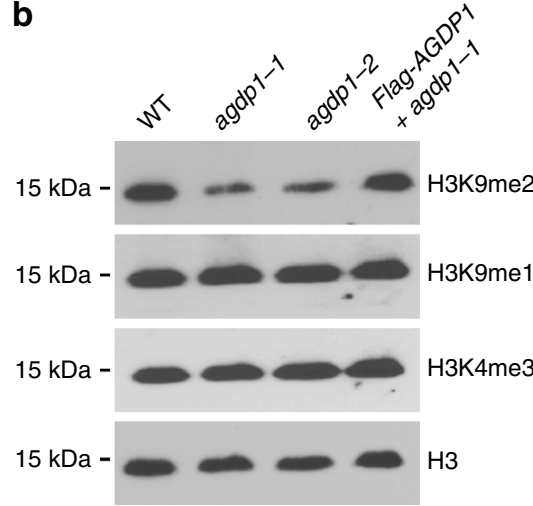

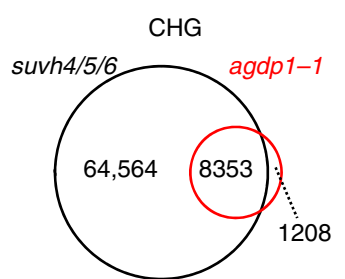
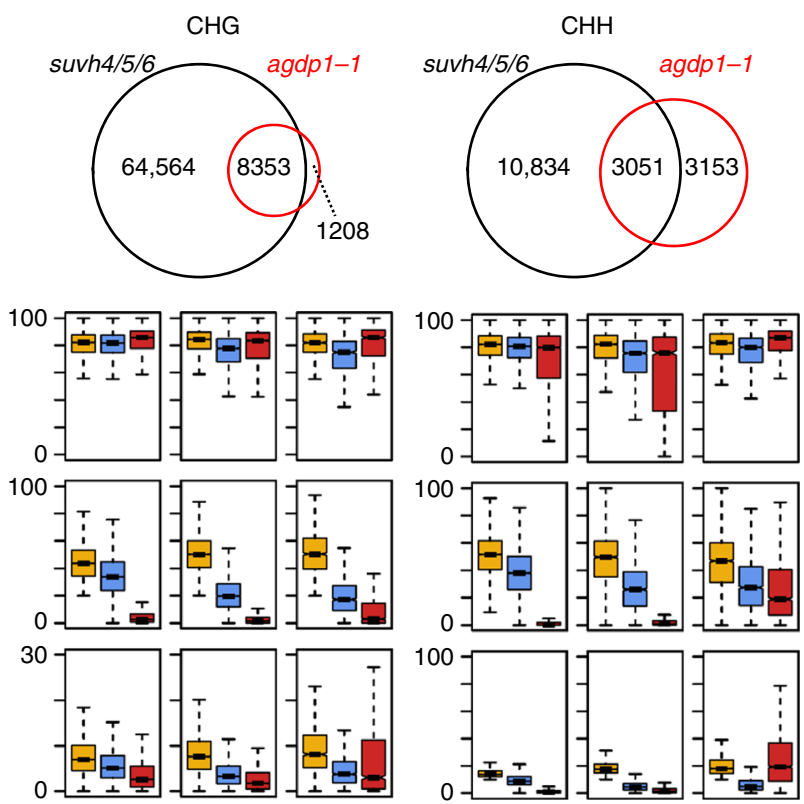

c
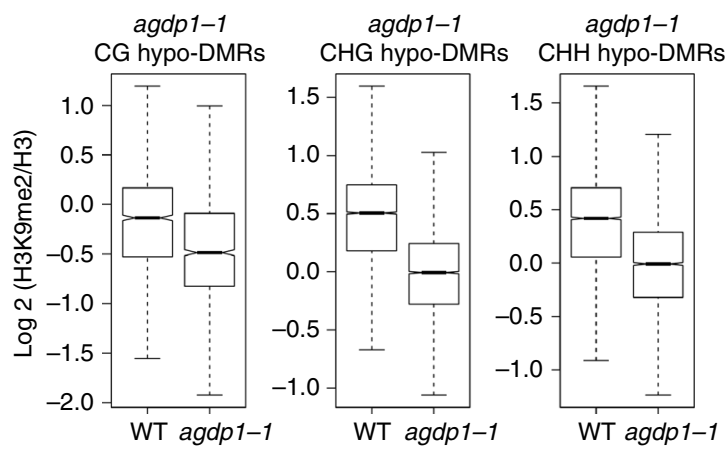

Fig. 5 agdp1 affects H3K9 dimethylation and suvh4/5/6-dependent CHG and CHH methylation. a Venn diagrams showing the overlap of hypo-DMRs between agdp1 and suvh4/5/6 in $\mathrm{CG}, \mathrm{CHG}$, and $\mathrm{CHH}$ contexts. Box plots represent $\mathrm{CG}, \mathrm{CHG}$, and $\mathrm{CHH}$ methylation levels of overlapping or unique hypoDMRs in the upper Venn diagrams. Yellow, blue, and red boxes indicate DNA methylation levels in the wild-type, agdp1-1, and suvh4/5/6, respectively. b Western blot analysis of $\mathrm{H} 3$ lysine methylation status in wild-type Col-0 plants and in agdp1-1, agdp1-2, and Flag-AGDP1 transgenic plants in the agdp1-1 mutant background with the antibodies specified on the right. c Box plots of levels of H3K9me2 relative to H3 in agdp1-1 CG, $\mathrm{CHG}$, and $\mathrm{CHH}$ hypo-DMRs. The $y$ axis represents the ChIP-seq read density normalized by $\mathrm{H} 3$ 
was reduced in suvh4/5/6, cmt2, drm1/2, and $d d m 1$ mutants (Supplementary Fig. 5).

To determine whether the agdp1 mutation affects $\mathrm{H} 3 \mathrm{~K} 9$ dimethylation levels, we investigated the global level of $\mathrm{H} 3 \mathrm{~K} 9 \mathrm{me} 2$ in the wild-type and in the agdp1-1 and agdp1-2 mutants with immunoblotting assays. The results showed that $\mathrm{H} 3 \mathrm{~K} 9 \mathrm{me} 2$ levels were reduced in agdp1-1 and agdp1-2, whereas the levels of H3K9me1 and H3K4me3 in agdp1-1 and agdp1-2 mutants were comparable to that in the wild-type (Fig. 5b). Moreover, the reduced $\mathrm{H} 3 \mathrm{~K} 9 \mathrm{me} 2$ level in agdp1-1 was fully rescued by the pAGDP1-Flag-AGDP1 transgene (Fig. 5b). To further confirm the reduction of $\mathrm{H} 3 \mathrm{~K} 9 \mathrm{me} 2$ levels in agdp1, we performed H3K9me2 and H3 ChIP-Seq in wild-type and agdp1-1. Box plot analysis showed that the H3K9me2 levels in agdp1-1 mutants are reduced at AGDP1-target sites (Fig. 5c). These results indicate that agdp1 affects SUVH4/5/6-dependent CHG and $\mathrm{CHH}$ methylation and has a global effect on H3K9 dimethylation. Our RNA-seq data showed that the expression levels of CMT2, CMT3, SUVH4, SUVH5, SUVH6, and DDM1 were not affected by mutation of agdp1-1 (Supplementary Data 2). This indicates that the reductions of non-CG methylation and of $\mathrm{H} 3 \mathrm{~K} 9 \mathrm{me} 2$ in agdp1 mutants is not caused by the reduced expression of these genes.

AGDP1 is enriched in heterochromatin. Subnuclear localization analyses showed that, the fluorescence of the control green fluorescent protein (GFP), was dispersed in the nuclear and excluded from the nucleolus regions, GFP-AGDP1 fluorescence showed similar localization patterns with 4,6-diamidino-2-phenylindole (DAPI) staining, except for the strong fluorescence signal within the nucleolus (Supplementary Fig. 6). To determine whether AGDP1 directly associates with heterochromatin, we performed ChIP assays with pAGDP1-Flag-AGDP1 transgenic plants. The transgene was functional in vivo, because its expression fully rescued the phenotypes of reduced H3K9me2 level and increased expression of $A t G P 1$ and AtMU1 in the agdp1-1 mutant (Fig. 5b and Supplementary Fig. 7). ChIP-qPCR results showed that AGDP1 is enriched at AtGP1 and AtMU1 loci but not at the negative control locus Actin2 (Fig. 6a). To further map the genome-wide occupancy of AGDP1, we carried out ChIP-seq analysis (ChIP followed by sequencing) for Flag-AGDP1 and wild-type control samples. The mapped reads at AtGP1 and AtMU1 loci showed that AGDP1 is enriched at these two TEs (Fig. 6b). The genome-wide analysis revealed a total of 1914 AGDP1 enrichment peaks (Supplementary Data 5). The distribution analysis throughout the five chromosomes showed that AGDP1 peaks are concentrated in centromeric and pericentromeric regions (Fig. 6c), which is similar to that of agdp1 CHG and $\mathrm{CHH}$ hypo-DMRs (Fig. 4d). To determine which histone marks are associated with AGDP1-binding peaks, we compared the AGDP1 occupancy profiles with published genome-wide histone modification data ${ }^{34,35}$. Compared with the randomly selected genomic regions with the same length distribution as the AGDP1 peaks, regions encompassing the AGDP1 peaks show a slight increase in the level of H3 (Fig. 6d), indicating a higher nucleosome density in AGDP1 targets. In addition, AGDP1 peaks were found to be positively associated with the heterochromatin marks H3K9me2 and H3K27me1 (Fig. 6d). In contrast, AGDP1 peaks were found to be negatively associated with most active histone marks compared with control regions (Fig. 6d). Consistently, Scatter plot analysis showed that AGDP1 enrichment is highly correlated with $\mathrm{H} 3 \mathrm{~K} 9 \mathrm{me} 2$ modification (Supplementary Fig. 8a). At AGDP1-binding regions, the $\mathrm{CHG}$ and $\mathrm{CHH}$ methylation and $\mathrm{H} 3 \mathrm{~K} 9 \mathrm{me} 2$ levels were decreased in agdp1-1 (Fig. 6e and Supplementary Fig. 8b). Snapshots of the genome browser showed that AGDP1 is enriched at four representative
TE regions and required both for the silencing and the non-CG methylation of these four TEs (Fig. $6 \mathrm{f}$ and Supplementary Fig. 9).

AGDP1 preferentially binds to long TEs. Our WGBS results showed that most of the CHG and CHH hypo-DMRs are located in TE regions (Fig. 4e). Similar to the distribution of CHG and CHH hypo-DMRs, most AGDP1-binding sites are in TE regions (Fig. 7a). We found that the average length of AGDP1-associated TEs was greater than the average length of all TEs in the genome (Fig. 7b). Further analysis showed that the enrichment of AGDP1 in long TEs is higher than that in short TEs (Fig. 7c and Supplementary Fig. 10). These results indicate that AGDP1 preferentially binds to long TEs. To investigate the correlation between AGDP1-binding profiles and AGDP1 influence on the methylation of TEs, we determined the relationship between average DNA methylation level and TE length. We found that the absolute levels of CHG and CHH methylation were more affected in long TEs than in short TEs in the agdp1-1 mutant (Fig. 7d). Based on these results, we conclude that AGDP1 preferentially binds to long TEs and thus affects their DNA methylation.

\section{Discussion}

Our structural studies suggested that the three pairs of tandem AGDs of AGDP1 can recognize a serials histone mark, including unmodified H3R2, H3K4, and H3R8, and dimethylated H3K9. $\mathrm{H} 3 \mathrm{~K} 4 \mathrm{me} 0$ and $\mathrm{H} 3 \mathrm{~K} 9 \mathrm{me} 2$ are specifically recognized by the two surface pockets of the AGD1 and AGD2, respectively (Figs. 2a, b). The two pockets are physically adjacent (Figs. 2a, b). However, $\mathrm{H} 3 \mathrm{~K} 4 \mathrm{me} 0$ and $\mathrm{H} 3 \mathrm{~K} 9 \mathrm{me} 2$ span six residues, such that the two pockets must be well separated if they are to properly capture both marks. The unique helical conformation of the H3K9me2 peptide can shrink the peptide, making the $\mathrm{H} 3 \mathrm{~K} 4 \mathrm{me} 0$ and H3K9me2 marks physically closer and fitting into the two adjacent pockets of AGD1 and AGD2, respectively (Figs. 2a, b). Therefore, the unique helical conformation of the peptide ensures the proper and simultaneous capture of the two marks in a dualrecognition mode.

The AGD12 of AGDP1 adopts a tandem tudor-like conformation (Fig. 1f). The tandem tudor has been extensively studied as a methylated histone reader module. Overall, the AGD12 structure resembles the human UHRF1 tandem tudor and Arabidopsis SHH1 SAWADEE domains, both of which function as $\mathrm{H} 3 \mathrm{~K} 9 \mathrm{me}$ readers with similar recognition mechanisms ${ }^{28,29,36-38}$. As shown in Fig. $2 \mathrm{~h}$, the SHH1 SAWADEE domain uses the longer edge of the two tudor domain interface to recognize the $\mathrm{H} 3 \mathrm{~K} 9 \mathrm{me} 2$ peptide, which is long enough to have two distant pockets that can simultaneously accommodate $\mathrm{H} 3 \mathrm{~K} 4 \mathrm{me} 0$ and $\mathrm{H} 3 \mathrm{~K} 9 \mathrm{me} 2$ (Fig. 2h). Therefore, the H3K9me2 peptide in the SAWADEE complex adopts an extended conformation (Fig. 2h). In contrast, the AGD12 of AGDP1 uses the shorter edge of the interface between the two AGDs to bind to the H3K9me2 peptide; as a result, the peptide must adopt a condensed conformation to allow the simultaneous positioning of the $\mathrm{H} 3 \mathrm{~K} 4 \mathrm{me} 0$ and $\mathrm{H} 3 \mathrm{~K} 9 \mathrm{me} 2$ marks into the two adjacent pockets (Fig. $2 \mathrm{~h}$ ). This mode of binding between AGDP1 and the H3K9me2 peptide has not been observed in other tandem tudorlike structures. Therefore, we conclude that the AGD12-H3K9me2 complex represents a different histonebinding interface on the tandem tudor/AGD-like readers.

Genome-wide mapping of AGDP1-binding sites by ChIP-seq showed that AGDP1 preferentially binds to long TEs. In the Arabidopsis genome, short TEs are enriched in chromosome arms, whereas long TEs are enriched in heterochromatin and are marked by $\mathrm{H} 3 \mathrm{~K} 9 \mathrm{me} 2^{39,40}$. Our in vitro binding data suggest that a single AGDP1 protein possesses three H3K9me2-binding sites 
a

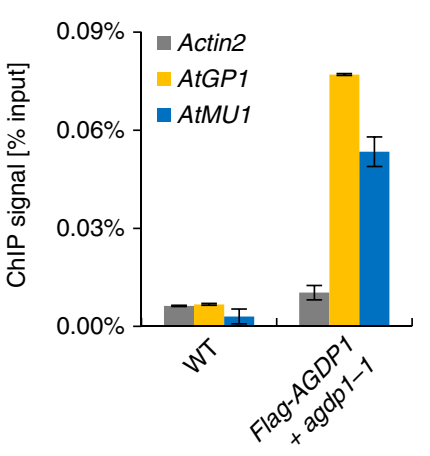

b

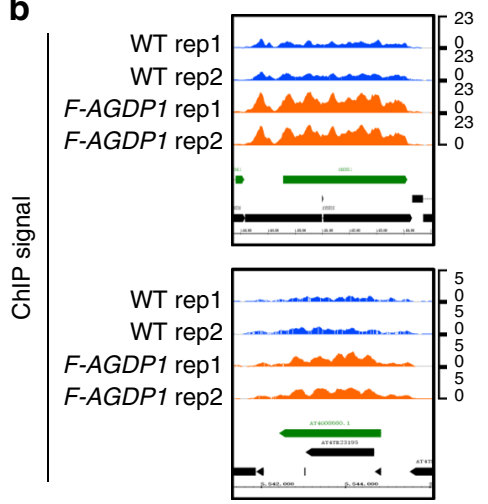

C

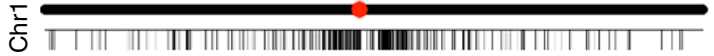

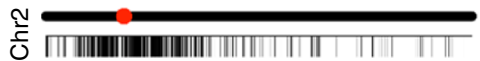

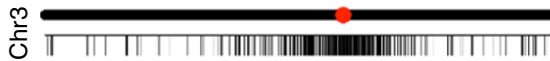

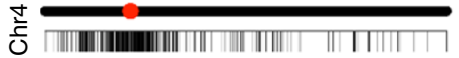

边

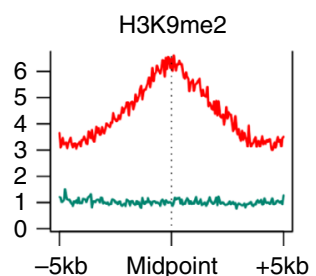

H3K9Ac

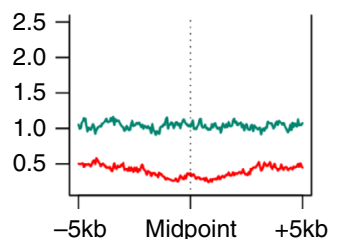

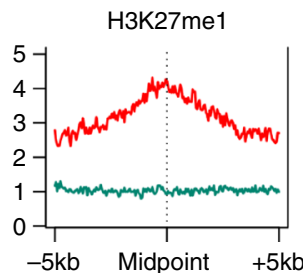

H3K18AC

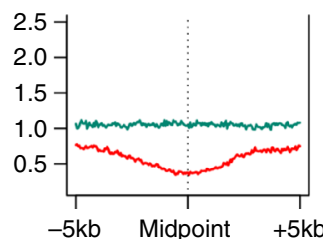

H3K27me3

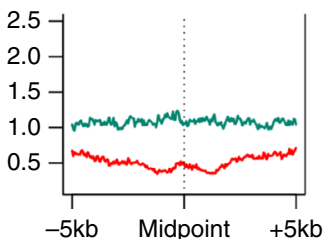

H3K36me2

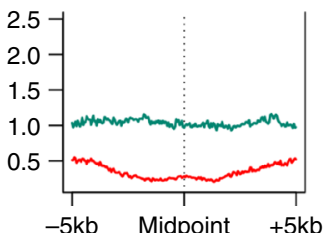

H3K4me2

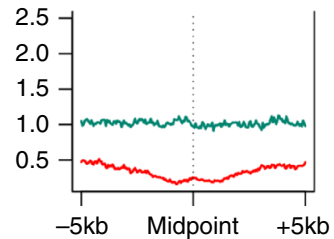

H3K36me3

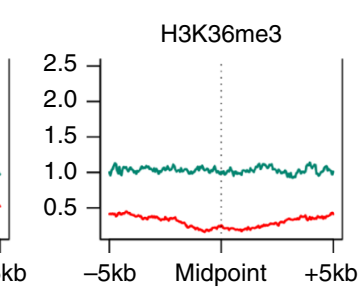

f $\left.\begin{array}{r}10 \\ 0 \\ 10 \\ 0 \\ 0 \\ 1 \\ -1 \\ 1 \\ -1 \\ 1 \\ 1 \\ -1 \\ 1 \\ -1 \\ 1 \\ -1 \\ 1 \\ -1 \\ 2 \\ 0 \\ 2 \\ 0 \\ 5 \\ 0 \\ 5 \\ 0\end{array}\right]$

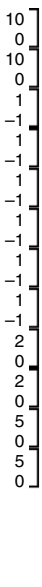

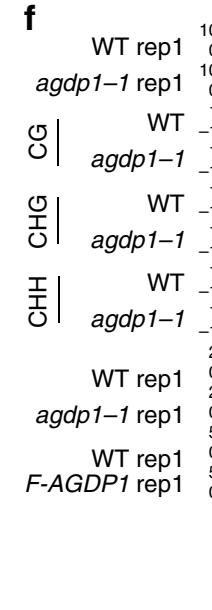

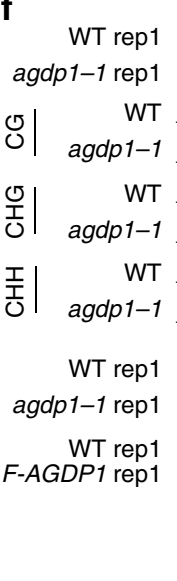

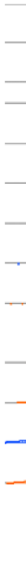

d

H3K4me3
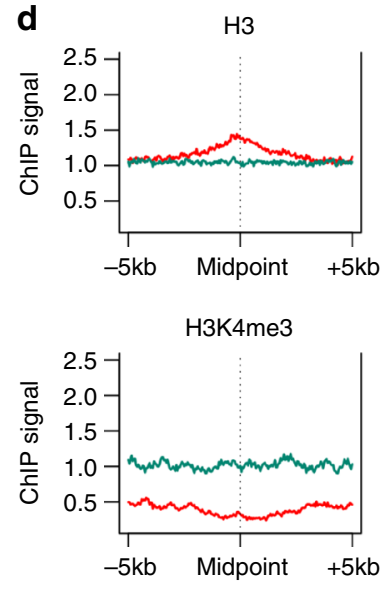

e

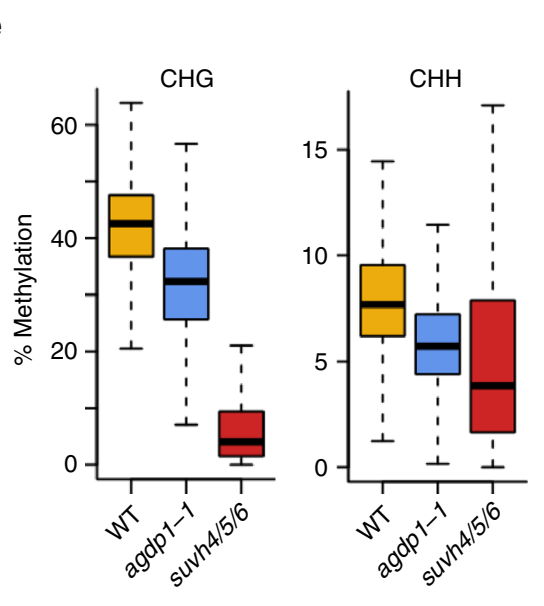

. 
a

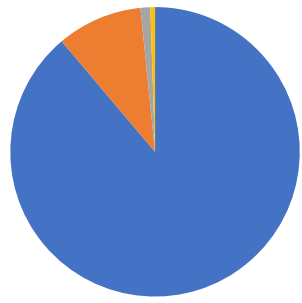

$\square \mathrm{TE}$

- Gene

- Intergenic

- Pseudogene+other b

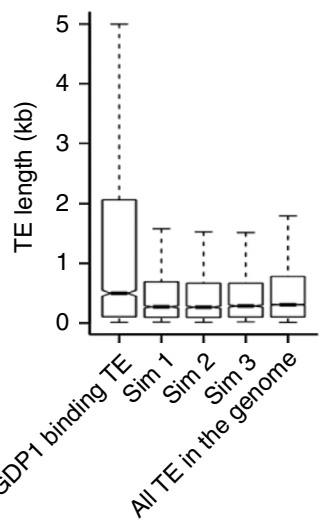

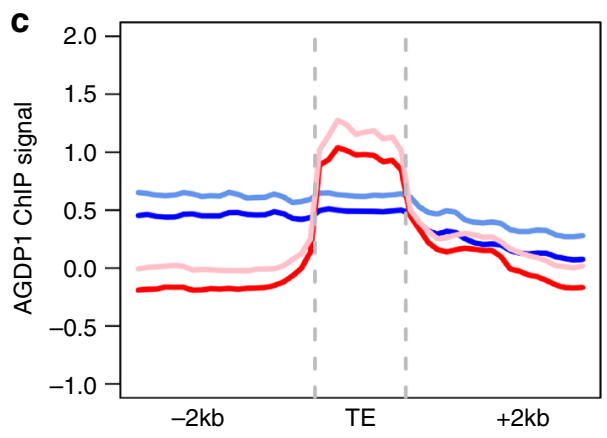

d
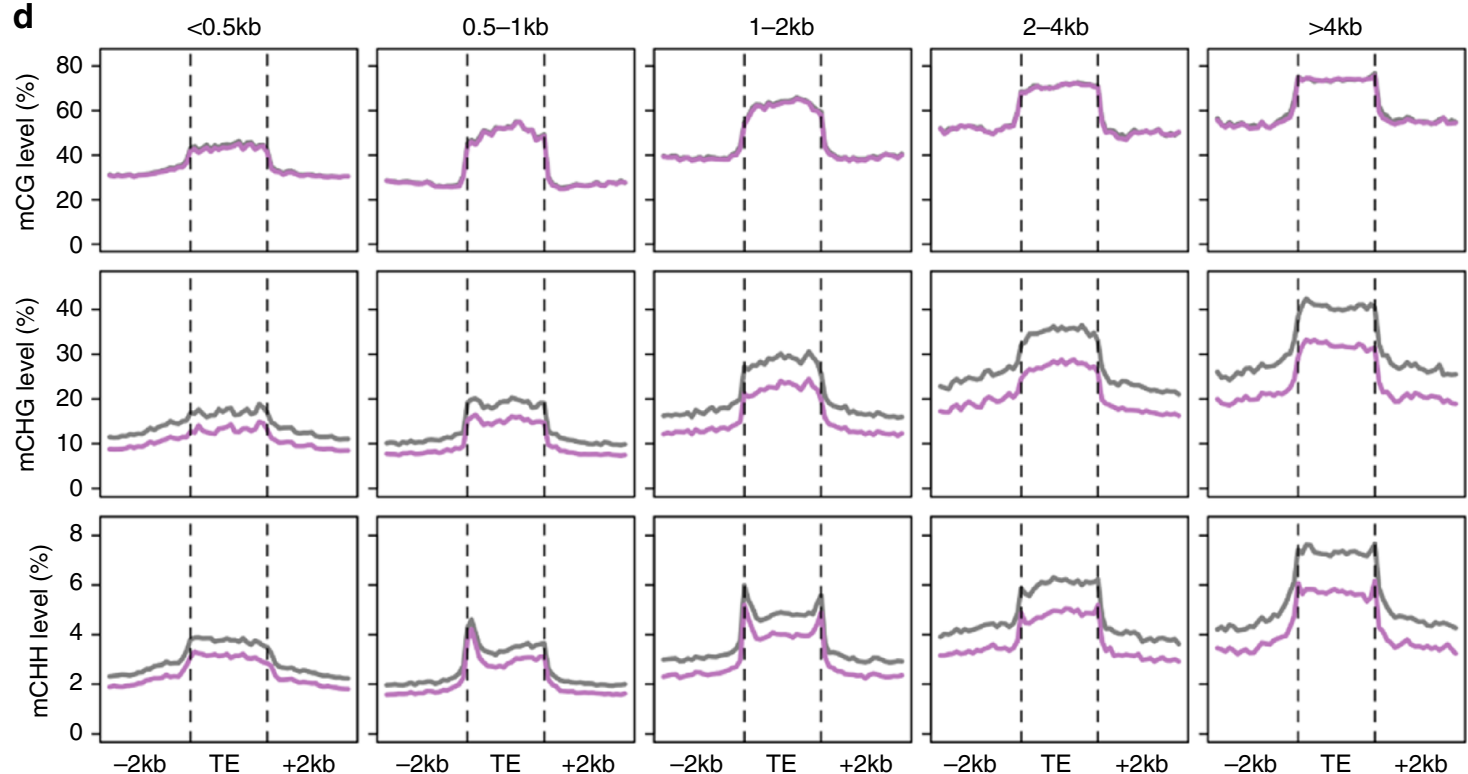

Fig. 7 AGDP1 preferentially binds to long TEs. a Composition of the genomic locations of the AGDP1-enriched peaks. TE, transposable element. b Box plots of the length of TEs that are bound to AGDP1 and of all TEs in the Arabidopsis genome. Only TEs with $>80 \%$ ovelap with ChIP-seq peaks were defined as "AGDP1 bound TEs". Three sets of randomly distributed peaks with the same length distribution of the AGDP1 peaks served as control regions. c Metaplot analysis of AGDP1 enrichment over long and short TEs. Blue and light blue curves represent ChIP signals of replicate 1 and 2 over short TEs (length $\leq 4 \mathrm{~kb}$ ). Red and light red curves represent ChIP signals of replicate 1 and 2 over long TEs (length $>4 \mathrm{~kb}$ ), respectively. d Average DNA methylation levels along different length of TEs in different cytosine contexts. Gray and pink curves represent DNA methylation levels of wild-type and agdp1-1, respectively

also demonstrated that AGDP1 is associated with heterochromatin and is required for transcriptional silencing, non-CG DNA methylation, and $\mathrm{H} 3 \mathrm{~K} 9$ dimethylation at some loci. The hypo-DMRs of agdp1 overlap with those of suvh4/5/6 and cmt2/ 3 , indicating that AGDP1 is functionally involved in the cyclic feedback loop between H3K9me2 and non-CG methylation. Both SUVH4/5/6 and CMT2/3 can directly couple the reading of an epigenetic silencing mark and the writing of another epigenetic silencing mark by themselves ${ }^{13,16}$. To identify AGDP1-associated factors, we performed immunoprecipitation followed by mass spectrometry (IP-MS) analysis with $p A G D P 1$ Flag-AGDP1 transgenic plants for four replicates, but did not identify SUVH4/5/6, CMT2/3, and DDM1 from the FlagAGDP1 IP samples (Supplemental Data 6). It is possible that AGDP1 does not directly recruit SUVH $4 / 5 / 6$, CMT2/3, or DDM1 but instead serves in a structural role, such as acting as a scaffold protein to bridge multiple nucleosome together. Our ChIP-seq data indicated that AGDP1 is preferentially located at long TEs. Previous studies showed that DDM1 allow methyltransferase access to nucleosomal templates and longer TEs that are blocked by the H1 linker ${ }^{40,41}$. It is still unknown why DDM1 preferentially affects the chromatin architecture of long TEs but not short TEs. We found a substantial overlap of hypo-DMRs between agdp 1 and $d d m 1$ mutants (Supplementary Fig. 4). We propose that the binding of AGDP1 to the heterochromatin mark $\mathrm{H} 3 \mathrm{~K} 9 \mathrm{me} 2$ might induce a change in chromatin structure and thereby create a permissive chromatin environment for DDM1 and subsequently for the DNA methyltransferases. It would be interesting to see if histone $h 1$ mutation can suppress agdp1 mutant phenotype by future studies. The enrichment of AGDP1 within the nucleolus indicates that AGDP1 may also have functions related to the nucleolus. The nucleolus is the site of $45 \mathrm{~S}$ pre-rRNA transcription, rRNA, tRNA, and endogenous nuclear siRNAs processing ${ }^{42,43}$. The preferential binding of AGDP1 to heterochromatin is consistent with the nucleolus-associated chromatin domains in A. thaliana being primarily genomic regions with heterochromatic signatures. These regions also include 
TEs and mostly inactive protein-coding genes ${ }^{44}$. Further studies are required to clarify the functions of AGDP1 in the nucleolus.

\section{Methods}

Plant materials and growth conditions. All plants were grown under a long-day photoperiod with $16 \mathrm{~h}$ of light at $22^{\circ} \mathrm{C}$ and $8 \mathrm{~h}$ of darkness at $20^{\circ} \mathrm{C}$. The Arabidopsis T-DNA insertion lines agdp1-1 (Salk_134878) and agdp1-2 (Salk_130936) were obtained from the Arabidopsis Biological Resource Center. The suvh4/5/6 mutant was obtained from J. Bender, Brown University ${ }^{11}$

To generate AGDP1 complementation lines, the 2172-bp promoter regions of $A G D P 1$ and AGDP1 genomic DNA were cloned into the modified pCAMBIA1305 vector (with a $3 \times$ Flag tag at the $\mathrm{N}$ terminus of AGDP1) using the In-Fusion HD Cloning Kit (Clontech, 639648). The construct was transformed into agdp1-1 mutants using Agrobacterium GV3101 and the standard floral dip method. The primers used for plasmids construction are listed in Supplementary Data 7.

Peptide affinity purification and mass spectrometric analysis. To extract the nuclear proteins for peptide affinity purification, $1 \mathrm{~g}$ of wild-type Arabidopsis flowers was harvested and ground in liquid nitrogen. The ground material was homogenized in $15 \mathrm{ml}$ of Honda Buffer (20 mM HEPES-KOH, pH 7.4, $0.44 \mathrm{M}$ sucrose, $1.25 \%$ ficoll, $2.5 \%$ Dextran T40, $10 \mathrm{mM} \mathrm{MgCl} 2,0.5 \%$ Triton X-100, $5 \mathrm{mM}$ DTT, $1 \mathrm{mM}$ phenylmethylsulfonyl fluoride (PMSF), Roche protease inhibitor cocktail) and filtered through two layers of Miracloth. The filtrates were centrifuged at $2000 \mathrm{~g}$ for $15 \mathrm{~min}$ at $4{ }^{\circ} \mathrm{C}$ to precipitate the nuclei. Nuclear pellets were washed two times with $1 \mathrm{ml}$ of Honda buffer and then were resuspended in $600 \mu \mathrm{l}$ of Nuclei Lysis Buffer (50 mM Tris-HCl pH 8.0, $150 \mathrm{mM} \mathrm{NaCl}, 1 \%$ NP40, $0.5 \mathrm{mM}$ DTT, 1 mM PMSF, Roche protease inhibitor cocktail). After sonication, nuclei extracts were centrifuged at $20,000 \mathrm{~g}$ for $10 \mathrm{~min}$ at $4{ }^{\circ} \mathrm{C}$. The supernatant was incubated with $2 \mu \mathrm{g}$ of biotinylated histone peptides and Streptavidin Magnetic Beads (Thermo Scientific, 88816) for $3 \mathrm{~h}$ with rotation at $4{ }^{\circ} \mathrm{C}$. Histone peptides $\mathrm{H} 3(12$ 403), H3K9me1 (12-569), H3K9me2 (12-430), and H3K9me3 (12-568) were purchased from EMD Millipore. After incubation, beads were washed three times with wash buffer 1 ( $320 \mathrm{mM} \mathrm{NaCl}, 50 \mathrm{mM}$ Tris- $\mathrm{HCl} \mathrm{pH}$ 8.0, 0.5\% NP40), and three times with wash buffer $2(150 \mathrm{mM} \mathrm{NaCl}, 50 \mathrm{mM}$ Tris- $\mathrm{HCl} \mathrm{pH}$ 8.0).

For LC-MS/MS analysis, IP beads with captured proteins were lysed in $12 \mathrm{mM}$ sodium deoxycholate (SDC)-sodium lauroyl sarcosinate (SLS): $100 \mathrm{mM}$ Tris-HCl (pH 8.5), $12 \mathrm{mM} \mathrm{SDC}$, and $12 \mathrm{mM}$ SLS. Proteins were reduced and alkylated with $10 \mathrm{mM}$ tris-(2-carboxyethyl) phosphine and $40 \mathrm{mM}$ chloroacetamide at $95{ }^{\circ} \mathrm{C}$ for 5 min. The supernatant containing reduced and alkylated proteins was transferred into a new Eppendorf tube and diluted fivefold with $50 \mathrm{mM}$ triethylammonium hydrogen carbonate buffer and digested with $500 \mathrm{ng}$ trypsin (Sigma) overnight. The SDC and SLS were removed by adding $100 \%$ acetyl acetate in a ratio $1: 1$ ( $\mathrm{vol} / \mathrm{vol})$ sample to acetyl acetate volume ratio. Samples were acidified with $10 \%$ trifluoroacetic acid to $\mathrm{pH} \sim 3$, and desalted by a custom-made stage tip with a styrene divinyl benzene (SDB-XC) membrane $(3 \mathrm{M})$. The dried peptides were resuspended in $30 \mu \mathrm{L}$ of $0.3 \%$ formic acid (FA) with $3 \%$ acetonitrile (ACN), quantified by BCA assay (Thermo Fisher Scientific), and injected into an Easy-nLC 1000 (Thermo Fisher Scientific). Peptides were separated on a $45 \mathrm{~cm}$ in-house packed column $(360 \mu \mathrm{m}$ OD $\times 75 \mu \mathrm{m}$ ID) containing C18 resin $(2.2 \mu \mathrm{m}, 100 \AA$, Michrom Bioresources) with a $30 \mathrm{~cm}$ column heater (Analytical Sales and Services) set at $60^{\circ} \mathrm{C}$. The mobile phase buffer consisted of $0.1 \% \mathrm{FA}$ in ultra-pure water (buffer A) with an eluting buffer of $0.1 \% \mathrm{FA}$ in $80 \% \mathrm{ACN}$ (buffer B) run over a linear $65 \mathrm{~min}$ gradient of $5 \%-30 \%$ buffer B at a flow rate of $250 \mathrm{~nL} / \mathrm{min}$. The EasynLC 1000 was coupled online with a Velos Pro LTQ-Orbitrap mass spectrometer (Thermo Fisher Scientific). The mass spectrometer was operated in the datadependent mode in which a full MS scan (from m/z 350-1500 with the resolution of 30,000 at $400 \mathrm{~m} / \mathrm{z}$ ) was followed by the 10 most intense ions being subjected to collision-induced dissociation (CID) fragmentation. CID fragmentation was performed and acquired in the linear ion trap (normalized collision energy $30 \%$, AGC 3e4, max injection time $100 \mathrm{~ms}$, isolation window $3 \mathrm{~m} / \mathrm{z}$, and dynamic exclusion $60 \mathrm{~s}$ ). The raw files were searched directly against Arabidopsis thaliana database (TAIR10) with no redundant entries using SEQUEST HT algorithm in Proteome Discoverer version 2.2 (Thermo Fisher Scientific). Peptide precursor mass tolerance was set at $15 \mathrm{ppm}$, and MS/MS tolerance was set to $0.6 \mathrm{Da}$. Search criteria included a variable modifications of oxidation $(+15.995 \mathrm{Da})$ on methionine residues, and acetylation $(+42.011 \mathrm{Da})$ at $\mathrm{N}$-terminus of protein, and a static carbamidomethylation of cysteines $(+57.021 \mathrm{Da})$. Search was performed with full tryptic digestion and allowed a maximum of two missed cleavages on the peptides analyzed from the sequence database. Relaxed and strict false discovery rates were set for 0.05 and 0.01 , respectively.

Protein expression and purification. The gene encoding AGD12 of RsAGDP1 (residues 26-180) was cloned into a self-modified pMBP-His vector to fuse $\mathrm{N}$ terminal hexahistidine plus an MBP tags to the target protein. The plasmids were transformed into Escherichia coli strain BL21(DE3) RIL. When the OD600 of the cell culture reached 0.7 , expression of the protein was induced at $20^{\circ} \mathrm{C}$ by adding isopropyl $\beta$-D-1-thiogalactopyranoside to a final concentration of $0.2 \mathrm{mM}$. The recombinant expressed protein was purified by a HisTrap column (GE Healthcare). The His-MBP tag was cleaved by tobacco etch virus protease and removed by a second step HisTrap column (GE Healthcare). The target protein was further purified using a Superdex G200 gel filtration column (GE Healthcare). AtAGDP1 AGD12 (residues 12-183), AGD34 (residues 198-364), AGD56 (residues 375-517), full-length AtAGDP1, RsAGDP1 AGD34 (residues 201-350), and AGD56 (residues 361-508) were cloned, expressed, and purified using the same protocol as used for RsAGDP1 AGD12. Because the RsAGDP1 AGD12 has no methionine residues, an L54M/L135M/L140M triple mutant was generated for producing Se-Met-substituted proteins. The Se-Met-substituted proteins were expressed in Se-Met containing M9 medium and were purified using the same protocol as used for the wild-type protein. All mutations were generated using a PCR-based method and were expressed and purified using the same protocol as used for the wild-type proteins. The peptides used in this study were purchased from the GL Biochem (Shanghai) Ltd.

Crystallization and structure determination. Before crystallization, the RsAGDP1 AGD12 was mixed with an H3(1-15)K9me2 peptide with a molar ratio of 1:4. The Se-Met-labeled RsAGDP1 AGD12 L54M/L135M/L140M triple mutant in complex with an $\mathrm{H} 3 \mathrm{~K} 9 \mathrm{me} 2$ peptide was crystallized in a condition of $15 \%$ PEG20000, 0.1 M MES, pH 6.5. The Se-Met-labeled AtAGDP1 AGD34 was crystallized in a condition of $3.5 \mathrm{M}$ sodium formate, $0.1 \mathrm{M}$ HEPES, pH 7.5. The crystals were cryo-protected in the reservoir solution supplemented with $15 \%$ glycerol and were flash cooled in liquid nitrogen. All data were collected at the beamline BL18U1 of the National Center for Protein Sciences Shanghai (NCPSS) at the Shanghai Synchrotron Radiation Facility (SSRF). The data were processed using the program HKL3000 suite ${ }^{45}$. The structure of RsAGDP1 AGD12-H3K9me2 complex was determined using the single-wavelength anomalous dispersion (SAD) method as implemented in the program Phenix ${ }^{46}$. The structure refinement and manual model building were carried out using the programs Phenix and Coot, respectivel $y^{46,47}$. Throughout the refinement, the geometry of the structure model was monitored using the program Molprobity ${ }^{48}$. The structure of AtAGDP1 AGD34 in free from was determined using the SAD method, too. The statistics for data collection and structure refinement are summarized in Supplementary Table 1.

ITC. The ITC-based binding experiments were carried out on a Microcal PEAQITC instrument (Malvern) at $25^{\circ} \mathrm{C}$. The protein samples were dialyzed overnight at $4{ }^{\circ} \mathrm{C}$. The protein samples were then diluted to $0.1 \mathrm{mM}$, and the lyophilized peptides were dissolved with the same buffer with a final concentration of $1.0-1.5 \mathrm{mM}$. The titration was performed with the standard protocol, and the data were fit using the program Origin 7.0.

RNA sequencing and data analysis. We performed RNA deep sequencing assay with five replicates for wild-type and agdp1-1, and three replicates for suvh4/5/6. Total RNA was extracted from $0.2 \mathrm{~g}$ of 10-day-old Arabidopsis seedlings with Trizol reagent (Invitrogen) and was sent to the Genomics Core Facilities of the PSC (Shanghai Center for Plant Stress Biology, Shanghai, China) for library preparation according to the manufacturer's (Illumina) instructions. The libraries were sequenced on an Illumina Hiseq 2500 platform. Reads were mapped to the TAIR10 genome using TopHat $2{ }^{49}$ with parameter "--b2-very-sensitive". The differential expression of protein-coding genes and TEs was identified with "Cuffdiff" program (with parameter "-u -b $<$ genome.fa $>$ ") of Cufflinks ${ }^{50}(P<0.05$; log2(fold-change) $>1)$.

WGBS and data analysis. Genomic DNA was extracted from 10-day-old seedlings using the DNeasy Plant Maxi Kit (Qiagen, 68163) and was sent to the Genomics Core Facilities of the PSC (Shanghai Center for Plant Stress Biology, Shanghai, China) for bisulfite treatment. Libraries were prepared for sequencing according to the manufacturer's (Illumina) instructions and were sequenced on an Illumina HiSeq 2500 platform. For data analysis, low-quality sequences $(q<20)$ were trimmed using trim in BRAT-BW ${ }^{51}$, and clean reads were mapped to the TAIR10 genome using BRAT-BW and allowing two mismatches. To remove potential PCR duplicates, the remove-dupl command of BRAT-BW was used. DNA hypomethylated regions were identified according to Stroud et al. ${ }^{15}$ with some modifications. In brief, the genome was divided into 100-bp bins. Fisher's exact test was performed for methylated versus unmethylated cytosines for each context, within each bin, with false discovery rates (FDRs) estimated using a Benjamini-Hochberg (BH) adjustment of Fisher's $P$-values calculated in the R environment. Bins with an absolute methylation difference of $0.4,0.2$, and 0.1 for CG, CHG, and $\mathrm{CHH}$, respectively, and with a $\mathrm{BH}$-corrected FDR $<0.01$ were selected. To avoid 100-bp bins with few cytosines, only bins with at least four corresponding cytosines that were covered by at least four reads in both the mutant and wild-type were retained. The WGBS experiments were performed with one replicate and the reduction of DNA methylation was confirmed by chop-PCR. Full scans of the agarose gels and western blots are shown in Supplementary Fig. 11.

ChIP assays. ChIP was performed following the instruction from a previously published study with minor modifications ${ }^{52}$. In brief, $1 \mathrm{~g}$ of 10 -day-old seedlings were cross-linked with $1 \%$ formaldehyde for 20 min under vacuum and ground into fine powder in liquid nitrogen. Chromatin was isolated and sheared into 200- 
to 500 -bp fragments by sonication. The sonicated chromatin was incubated with 2 $\mu \mathrm{g}$ of anti-H3K9me2 (Abcam, ab1220), anti-H3 (Abcam, ab1791), or anti-Flag (Sigma, F1804) antibody and with $25 \mu \mathrm{l}$ of Dynabeads Protein G (Invitrogen, 10003D) for $5 \mathrm{~h}$ with rotation at $4{ }^{\circ} \mathrm{C}$. The precipitated chromatin DNA was then recovered and purified with a standard phenol-chloroform method. ChIP-qPCR was performed and results were calculated as percentage of input DNA. Sequences for the primers used for ChIP-qPCR are listed in Supplementary Data 7.

ChIP-seq and data analysis. Two biological replicates were prepared and sequenced for each ChIP-seq experiment. For ChIP-seq, DNA from three ChIPs was pooled to ensure enough DNA for library construction. ChIP samples were sent to the Genomics Core Facilities of the PSC (Shanghai Center for Plant Stress Biology, Shanghai, China) for library construction and Illumina sequencing. ChIPseq reads were mapped to the TAIR10 genome with Bowtie $2^{53}$ with parameter "- $\mathrm{k}$ 10 --very-sensitive --no-unal --no-mixed --no-discordant" and PCR duplicates were removed with "samtools rmdup" 54 . AGDP1-binding peaks were identified with $\operatorname{SICER}^{55}$ (parameter: ["redundancy threshold" = 1] ["window size (bp)" = 200] ["fragment size" = 200] ["effective genome fraction" = 0.84] ["gap size (bp)" $=600]$ ["FDR" $=0.05]$ ). AGDP1 peaks associated histone features were analyzed as previously described ${ }^{35}$. The random peaks with the same length distribution is generated by "shuffle" command from the BEDTools suite.

AGDP1 nuclear localization. 35S::GFP was cloned into the modified $p C A M$ BIA1305 vector, which was named $p$ CAMBIA1305-35S::GFP. To determine the nuclear localization of AGDP1, the coding sequence of AGDP1 was amplified and cloned into the PCAMBIA1305-35S::GFP vector with its $\mathrm{N}$-terminal tagged by GFP. Leaves of tobacco plants ( $N$. benthamiana) were infiltrated with agrobacterium GV3101 strain carrying $p$ CAMBIA1305-35S::GFP or $p$ CAMBIA1305-35S::GFPAGDP1 plasmids. After 2 days, GFP-AGDP1 and GFP control fluorescence were observed in leaves of tobacco plants with a Zeiss LSM 880 upright confocal microscope.

Analysis of RNA transcripts at individual loci. Total RNA was extracted from 10-day-old seedlings with Trizol reagent (Invitrogen, 15596026). After contaminated DNA was removed by the DNA-free Kit (Invitrogen, AM1907), $1 \mu \mathrm{g}$ of total RNA was used for RT using both oligo dT and random primers. Real-time PCR was performed on a CFX96 real-time PCR detection system (Bio-Rad) using iTaq Universal SYBR Green Supermix (Bio-Rad, 1725122). The actin gene ACT7 was amplified as an internal control. The results presented were based on at least three biological replications. All primer sequences are listed in Supplementary Data 7.

\section{Data availability}

All high-throughput sequencing data generated in this study have been deposited in GEO with accession codes GSE111609. X-ray structures have been deposited in the RCSB Protein Data Bank with accession codes 5ZWX for RsAGDP1 AGD12H3K9me2 complex and 5ZWZ for AtAGDP34. All other data are available from the corresponding authors on request.

Received: 2 April 2018 Accepted: 4 October 2018

Published online: 31 October 2018

\section{References}

1. Campos, E. I. \& Reinberg, D. Histones: annotating chromatin. Annu. Rev. Genet. 43, 559-599 (2009).

2. Bannister, A. J. \& Kouzarides, T. Regulation of chromatin by histone modifications. Cell Res. 21, 381-395 (2011).

3. Liu, C., Lu, F., Cui, X. \& Cao, X. Histone methylation in higher plants. Annu. Rev. Plant. Biol. 61, 395-420 (2010).

4. Bernatavichute, Y. V., Zhang, X., Cokus, S., Pellegrini, M. \& Jacobsen, S. E. Genome-wide association of histone $\mathrm{H} 3$ lysine nine methylation with CHG DNA methylation in Arabidopsis thaliana. PLoS. ONE. 3, e3156 (2008).

5. Stroud, H. et al. Non-CG methylation patterns shape the epigenetic landscape in Arabidopsis. Nat. Struct. Mol. Biol. 21, 64-72 (2014)

6. Du, J., Johnson, L. M., Jacobsen, S. E. \& Patel, D. J. DNA methylation pathways and their crosstalk with histone methylation. Nat. Rev. Mol. Cell Biol. 16, 519-532 (2015).

7. Zhang, X. et al. Genome-wide high-resolution mapping and functional analysis of DNA methylation in arabidopsis. Cell 126, 1189-1201 (2006).

8. Cokus, S. J. et al. Shotgun bisulphite sequencing of the Arabidopsis genome reveals DNA methylation patterning. Nature 452, 215-219 (2008).

9. Lister, R. et al. Highly integrated single-base resolution maps of the epigenome in Arabidopsis. Cell 133, 523-536 (2008).
10. Jackson, J. P., Lindroth, A. M., Cao, X. \& Jacobsen, S. E. Control of CpNpG DNA methylation by the KRYPTONITE histone $\mathrm{H} 3$ methyltransferase. Nature 416, 556-560 (2002).

11. Ebbs, M. L. \& Bender, J. Locus-specific control of DNA methylation by the Arabidopsis SUVH5 histone methyltransferase. Plant Cell 18, 1166-1176 (2006).

12. Malagnac, F., Bartee, L. \& Bender, J. An Arabidopsis SET domain protein required for maintenance but not establishment of DNA methylation. EMBO J. 21, 6842-6852 (2002).

13. $\mathrm{Du}$, J. et al. Mechanism of DNA methylation-directed histone methylation by KRYPTONITE. Mol. Cell 55, 495-504 (2014).

14. Li, X. et al. Mechanistic insights into plant SUVH family H3K9 methyltransferases and their binding to context-biased non-CG DNA methylation. Proc. Natl. Acad. Sci. USA 115, E8793-E8802 (2018).

15. Stroud, H., Greenberg, M. V., Feng, S., Bernatavichute, Y. V. \& Jacobsen, S. E. Comprehensive analysis of silencing mutants reveals complex regulation of the Arabidopsis methylome. Cell 152, 352-364 (2013).

16. Du, J. et al. Dual binding of chromomethylase domains to H3K9me2containing nucleosomes directs DNA methylation in plants. Cell 151, 167-180 (2012).

17. Law, J. A. \& Jacobsen, S. E. Establishing, maintaining and modifying DNA methylation patterns in plants and animals. Nat. Rev. Genet. 11, 204-220 (2010).

18. Patel, D. J. \& Wang, Z. Readout of epigenetic modifications. Annu. Rev. Biochem. 82, 81-118 (2013).

19. Liu, R., Li, X., Chen, W. \& Du, J. Structure and mechanism of plant histone mark readers. Sci. China Life Sci. 61, 170-177 (2018).

20. Maurer-Stroh, S. et al. The tudor domain 'Royal Family': tudor, plant Agenet, Chromo, PWWP and MBT domains. Trends Biochem. Sci. 28, 69-74 (2003).

21. Lu, R. \& Wang, G. G. Tudor: a versatile family of histone methylation 'readers'. Trends Biochem. Sci. 38, 546-555 (2013).

22. Alpatov, R. et al. A chromatin-dependent role of the fragile $\mathrm{X}$ mental retardation protein FMRP in the DNA damage response. Cell 157, 869-881 (2014).

23. Ramos, A. et al. The structure of the $\mathrm{N}$-terminal domain of the fragile $\mathrm{X}$ mental retardation protein: a platform for protein-protein interaction. Structure 14, 21-31 (2006).

24. Myrick, L. K., Hashimoto, H., Cheng, X. \& Warren, S. T. Human FMRP contains an integral tandem Agenet (Tudor) and $\mathrm{KH}$ motif in the amino terminal domain. Hum. Mol. Genet. 24, 1733-1740 (2015).

25. Lei, M. et al. Arabidopsis EDM2 promotes IBM1 distal polyadenylation and regulates genome DNA methylation patterns. Proc. Natl. Acad. Sci. USA 111, 527-532 (2014)

26. Bortoluzzi, A., Amato, A., Lucas, X., Blank, M. \& Ciulli, A. Structural basis of molecular recognition of helical histone $\mathrm{H} 3$ tail by PHD finger domains. Biochem. J. 474, 1633-1651 (2017).

27. Li, J. et al. Structural basis for specific binding of human MPP8 chromodomain to histone $\mathrm{H} 3$ methylated at lysine 9. PLoS. One. 6, e25104 (2011).

28. Arita, K. et al. Recognition of modification status on a histone $\mathrm{H} 3$ tail by linked histone reader modules of the epigenetic regulator UHRF1. Proc. Natl. Acad. Sci. USA 109, 12950-12955 (2012).

29. Cheng, J. et al. Structural insight into coordinated recognition of trimethylated histone $\mathrm{H} 3$ lysine 9 (H3K9me3) by the plant homeodomain (PHD) and tandem tudor domain (TTD) of UHRF1 (ubiquitin-like, containing PHD and RING finger domains, 1) protein. J. Biol. Chem. 288, 1329-1339 (2013).

30. Chang, Y., Horton, J. R., Bedford, M. T., Zhang, X. \& Cheng, X. Structural insights for MPP8 chromodomain interaction with histone $\mathrm{H} 3$ lysine 9: potential effect of phosphorylation on methyl-lysine binding. J. Mol. Biol. 408, 807-814 (2011)

31. Kuo, A. J. et al. The BAH domain of ORC1 links H4K20me2 to DNA replication licensing and Meier-Gorlin syndrome. Nature 484, 115-119 (2012).

32. Panda, K. et al. Full-length autonomous transposable elements are preferentially targeted by expression-dependent forms of RNA-directed DNA methylation. Genome Biol. 17, 170 (2016).

33. Zhang, Q. et al. The chromatin remodeler DDM1 promotes hybrid vigor by regulating salicylic acid metabolism. Cell Discov. 2, 16027 (2016).

34. Luo, C. et al. Integrative analysis of chromatin states in Arabidopsis identified potential regulatory mechanisms for natural antisense transcript production. Plant J. 73, 77-90 (2013).

35. Tang, K., Lang, Z., Zhang, H. \& Zhu, J. K. The DNA demethylase ROS1 targets genomic regions with distinct chromatin modifications. Nat. Plants 2, 16169 (2016)

36. Holm, L. \& Rosenstrom, P. Dali server: conservation mapping in 3D. Nucleic Acids Res. 38, W545-W549 (2010).

37. Nady, N. et al. Recognition of multivalent histone states associated with heterochromatin by UHRF1 protein. J. Biol. Chem. 286, 24300-24311 (2011). 
38. Law, J. A. et al. Polymerase IV occupancy at RNA-directed DNA methylation sites requires SHH1. Nature 498, 385-389 (2013).

39. Ibarra, C. A. et al. Active DNA demethylation in plant companion cells reinforces transposon methylation in gametes. Science 337, 1360-1364 (2012).

40. Zemach, A. et al. The Arabidopsis nucleosome remodeler DDM1 allows DNA methyltransferases to access H1-containing heterochromatin. Cell 153, 193-205 (2013).

41. Lyons, D. B. \& Zilberman, D. DDM1 and Lsh remodelers allow methylation of DNA wrapped in nucleosomes. eLife 6, e30674 (2017).

42. Pontes, $\mathrm{O}$. et al. The Arabidopsis chromatin-modifying nuclear siRNA pathway involves a nucleolar RNA processing center. Cell 126, 79-92 (2006).

43. Earley, K. W. et al. Mechanisms of HDA6-mediated rRNA gene silencing: suppression of intergenic Pol II transcription and differential effects on maintenance versus siRNA-directed cytosine methylation. Genes Dev. $\mathbf{2 4}$ 1119-1132 (2010).

44. Pontvianne, F. et al. Identification of nucleolus-associated chromatin domains reveals a role for the nucleolus in $3 \mathrm{D}$ organization of the $A$. thaliana genome. Cell Rep. 16, 1574-1587 (2016).

45. Otwinowski, Z. \& Minor, W. Processing of X-ray diffraction data collected in oscillation mode. Methods Enzymol. 276, 307-326 (1997).

46. Adams, P. D. et al. PHENIX: a comprehensive Python-based system for macromolecular structure solution. Acta Crystallogr. D Biol. Crystallogr. 66, 213-221 (2010).

47. Emsley, P., Lohkamp, B., Scott, W. G. \& Cowtan, K. Features and development of Coot. Acta Crystallogr. D. Biol. Crystallogr. 66, 486-501 (2010).

48. Chen, V. B. et al. MolProbity: all-atom structure validation for macromolecular crystallography. Acta Crystallogr. D. Biol. Crystallogr. 66 12-21 (2010).

49. Kim, D. et al. TopHat2: accurate alignment of transcriptomes in the presence of insertions, deletions and gene fusions. Genome Biol. 14, R36 (2013).

50. Trapnell, C. et al. Differential analysis of gene regulation at transcript resolution with RNA-seq. Nat. Biotechnol. 31, 46-53 (2013).

51. Harris, E. Y., Ponts, N., Le Roch, K. G. \& Lonardi, S. BRAT-BW: efficient and accurate mapping of bisulfite-treated reads. Bioinformatics 28, 1795-1796 (2012).

52. Zhang, C. J. et al. The Arabidopsis acetylated histone-binding protein BRAT1 forms a complex with BRP1 and prevents transcriptional silencing. Nat. Commun. 7, 11715 (2016)

53. Langmead, B. \& Salzberg, S. L. Fast gapped-read alignment with Bowtie 2. Nat. Methods 9, 357-359 (2012)

54. Li, H. et al. The sequence alignment/map format and SAMtools. Bioinformatics 25, 2078-2079 (2009).

55. Zang, C. et al. A clustering approach for identification of enriched domains from histone modification ChIP-Seq data. Bioinformatics 25, 1952-1958 (2009).

\section{Acknowledgements}

We thank the staff at beamline BL18U1 of the National Center for Protein Sciences Shanghai (NCPSS) at the Shanghai Synchrotron Radiation Facility (SSRF) for assistance with data collection, and Prof. Judith Bender of Brown University, USA, for kindly providing the suvh $4 / 5 / 6$ mutant seeds. This work was supported by the National Key R\&D Program of China (2016YFA0503200 to J.D. and 2016YFA0500801 to X.-J.H.), the National Natural Science Foundation of China (31622032 and 31770782 to J.D.), the Chinese Academy of Sciences (to J.D. and J.-K.Z.), and the Youth Innovation Promotion Association CAS (to C.Z.).

\section{Author contributions}

C.Z., J.D., and J.-K.Z. conceived and designed the research. C.Z., L.P., P.Z., Y.J., H.Z., H. W., X.W., and F.W. performed peptide affinity purification, LC-MS/MS, and functional studies in Arabidopsis. X.D., Z.Y., J.L., and J.D. performed structure and biochemistry experiments. K.T. performed bioinformatics analyses. C.Z., X.D., W.A.T., X.-J.H., H.Z. R.A.B., J.D., and J.-K.Z. analyzed the data. C.Z., X.D., J.D., and J.-K.Z. wrote the manuscript.

\section{Additional information}

Supplementary Information accompanies this paper at https://doi.org/10.1038/s41467 018-06965-w.

Competing interests: The authors declare no competing interests.

Reprints and permission information is available online at http://npg.nature.com/ reprintsandpermissions/

Publisher's note: Springer Nature remains neutral with regard to jurisdictional claims in published maps and institutional affiliations.

Open Access This article is licensed under a Creative Commons Attribution 4.0 International License, which permits use, sharing, adaptation, distribution and reproduction in any medium or format, as long as you give appropriate credit to the original author(s) and the source, provide a link to the Creative Commons license, and indicate if changes were made. The images or other third party material in this article are included in the article's Creative Commons license, unless indicated otherwise in a credit line to the material. If material is not included in the article's Creative Commons license and your intended use is not permitted by statutory regulation or exceeds the permitted use, you will need to obtain permission directly from the copyright holder. To view a copy of this license, visit http://creativecommons.org/ licenses/by/4.0/

(c) The Author(s) 2018 